\title{
Asymptotic behavior of the nonlinear Schrödinger equation on exterior domain
}

\author{
Zhen-Hu Ning*
}

Abstract We consider the following nonlinear Schrödinger equation on exterior domain.

$$
\left\{\begin{array}{l}
i u_{t}+\Delta_{g} u+i a(x) u-|u|^{p-1} u=0 \quad(x, t) \in \Omega \times(0,+\infty) \\
\left.u\right|_{\Gamma}=0 \quad t \in(0,+\infty) \\
u(x, 0)=u_{0}(x) \quad x \in \Omega
\end{array}\right.
$$

where $1<p<\frac{n+2}{n-2}, \Omega \subset \mathbb{R}^{n}(n \geq 3)$ is an exterior domain and $\left(\mathbb{R}^{n}, g\right)$ is a complete Riemannian manifold. We establish Morawetz estimates for the system (1) without dissipation $(a(x) \equiv 0$ in (1) $)$ and meanwhile prove exponential stability of the system (11) with a dissipation effective on a neighborhood of the infinity.

It is worth mentioning that our results are different from the existing studies. First, Morawetz estimates for the system (1) are directly derived from the metric $g$ and are independent on the assumption of an (asymptotically) Euclidean metric. In addition, we not only prove exponential stability of the system (1) with non-uniform energy decay rate, which is dependent on the initial data, but also prove exponential stability of the system (11) with uniform energy decay rate. The main methods are the development of Morawetz multipliers in non (asymptotically) Euclidean spaces and compactness-uniqueness arguments.

Keywords nonlinear Schrödinger equation, Morawetz estimates, exponential stability, non (asymptotically) Euclidean spaces.

\section{Mathematics Subject Classification 58J45,93D20}

\footnotetext{
${ }^{*}$ Corresponding author, E-mail address: nzh41034@163.com

Zhen-Hu Ning, Faculty of Information Technology, Beijing University of Technology, Beijing, 100124, China. E-mail address: nzh41034@163.com.

This work is supported by the National Science Foundation of China, grants no.61473126 and no.61573342, and Key Research Program of Frontier Sciences, CAS, no. QYZDJ-SSW-SYS011.
} 


\section{Contents}

1 Introduction $\quad 2$

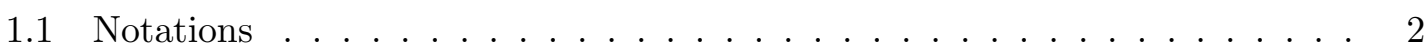

1.2 Nonlinear Schrödinger equation . . . . . . . . . . . . . . . 3

2 Main results

2.1 Well-posedness . . . . . . . . . . . . . . . . . . . 5

2.2 Morawetz estimates for the nonlinear Schrödinger equation in non (asymptotically) Euclidean spaces . . . . . . . . . . . 5

2.3 Stability of the damped nonlinear Schrödinger equation with non-uniform energy decay rate ....................... 7

2.4 Stability of the damped nonlinear Schrödinger equation with uniform energy decay rate ............................... 9

3 Multiplier Identities and Key Lemmas 9

4 Proofs of Morawetz estimates in non (asymptotically) Euclidean spaces 13

5 Proofs of stability with non-uniform energy decay rate 16

6 Proofs of stability with uniform energy decay rate 23

A Appendix: Proofs of Assumption (U1) and Assumption (U2) under a \begin{tabular}{l|l} 
strong geometric condition & 27
\end{tabular}

\section{Introduction}

\section{$1.1 \quad$ Notations}

Let $O$ be the origin of $\mathbb{R}^{n}(n \geq 3)$ and

$$
r(x)=|x|, \quad x \in \mathbb{R}^{n}
$$

be the standard distance function of $\mathbb{R}^{n}$. Moreover, let $\langle\cdot, \cdot\rangle$, div $, \nabla, \Delta$ and $I_{n}=\left(\delta_{i, j}\right)_{n \times n}$ be the standard inner product of $\mathbb{R}^{n}$, the standard divergence operator of $\mathbb{R}^{n}$, the standard gradient operator of $\mathbb{R}^{n}$, the standard Laplace operator of $\mathbb{R}^{n}$ and the unit matrix,respectively.

Suppose that $\left(\mathbb{R}^{n}, g\right)$ is a smooth complete Riemannian manifold with

$$
g=\sum_{i, j=1}^{n} g_{i j}(x) d x_{i} d x_{j}, \quad x \in \mathbb{R}^{n} .
$$

Let

$$
G(x)=\left(g_{i j}(x)\right)_{n \times n}, \quad x \in \mathbb{R}^{n} .
$$


Denote

$$
\langle X, Y\rangle_{g}=\langle G(x) X, Y\rangle, \quad|X|_{g}^{2}=\langle X, X\rangle_{g}, \quad X, Y \in \mathbb{R}_{x}^{n}, x \in \mathbb{R}^{n} .
$$

Let $D$ be the Levi-Civita connection of the metric $g$ and $H$ be a vector field, then the covariant differential $D H$ of the vector field $H$ is a tensor field of rank 2 as follow:

$$
D H(X, Y)(x)=\left\langle D_{Y} H, X\right\rangle_{g}(x) \quad X, Y \in \mathbb{R}_{x}^{n}, x \in \mathbb{R}^{n} .
$$

Let $S(r)$ be the sphere in $\mathbb{R}^{n}$ with radius $r$. Then

$$
\left\langle X, \frac{\partial}{\partial r}\right\rangle=0, \quad \text { for } \quad X \in S(r)_{x}, x \in \mathbb{R}^{n} \backslash O .
$$

Finally, we set $\operatorname{div} g, \nabla_{g}$ and $\Delta_{g}$ as the divergence operator of $\left(\mathbb{R}^{n}, g\right)$, the gradient operator of $\left(\mathbb{R}^{n}, g\right)$ and the Laplace-Beltrami operator of $\left(\mathbb{R}^{n}, g\right)$, respectively.

\subsection{Nonlinear Schrödinger equation}

Let $\Omega \subset \mathbb{R}^{n}$ be an exterior domain with smooth compact boundary $\Gamma$ and let $\nu(x)$ be the unit normal vector outside $\Omega$ in $\left(\mathbb{R}^{n}, g\right)$ for $x \in \Gamma$. Assume that the origin $O \notin \bar{\Omega}$. Denote

$$
d_{1}=\inf _{x \in \Gamma}|x| \quad \text { and } \quad d_{2}=\sup _{x \in \Gamma}|x| .
$$

Then $d_{2} \geq d_{1}>0$. For any constant $h>d_{2}$, we define

$$
\Omega(h)=\{x|x \in \Omega,| x \mid \leq h\}
$$

We consider the following system:

$$
\left\{\begin{array}{l}
i u_{t}+\Delta_{g} u+i a(x) u-|u|^{p-1} u=0 \quad(x, t) \in \Omega \times(0,+\infty) \\
\left.u\right|_{\Gamma}=0 \quad t \in(0,+\infty) \\
u(x, 0)=u_{0}(x) \quad x \in \Omega
\end{array}\right.
$$

where

$$
1<p<\frac{n+2}{n-2}
$$

and $a(x) \in C^{2}(\bar{\Omega})$ is a nonnegative real function satisfying

$$
\sup _{x \in \bar{\Omega}}\left(a(x)+\left|\nabla_{g} a(x)\right|_{g}+\left|\Delta_{g} a(x)\right|\right)<+\infty .
$$

Define the energy of the system (1.9) by

$$
E(t)=\frac{1}{2} \int_{\Omega}\left(|u|^{2}+\left|\nabla_{g} u\right|_{g}^{2}\right) d x_{g}+\frac{1}{p+1} \int_{\Omega}|u|^{p+1} d x_{g},
$$

where

$$
d x_{g}=\sqrt{\operatorname{det}(G(x))} d x, \quad|u|^{2}=u \bar{u}, \quad\left|\nabla_{g} u\right|_{g}^{2}=\left\langle\nabla_{g} u, \nabla_{g} \bar{u}\right\rangle_{g}
$$


For the free Schrödinger equation on a Riemannian manifold, many Strichartz estimates and local energy estimates are given by [6, 8, ,9, 11, 12, 23, 30, 44, 49, 53, 54] under the non-trapping assumption and the assumption of an Euclidean metric at infinity. There exists a wealth of literature on such estimates for the wave equation (see [10, 29, 39, 47, 48, 52, and references therein).

For the linear damped Schrödinger equation on Riemannian manifolds, the local energy decay in an exterior domain has been proved in [1, 2, 3, 4, 13, 35, 51] and many others under the geometric control condition (see [5, 50]). Under the non-trapping condition on an exterior domain, exponential decay for the global energy has been proved in [7] for the Schrödinger equation with a dissipation effective on a neighborhood of the infinity. For the nonlinear damped Schrödinger equation on compact manifold or Euclidean space, many stability results are given by [14, 15, 17, 18, 19, 20, 21, 25] and references therein. Such results are also based on the non-trapping assumption or geometric control condition.

The non-trapping assumption and geometric control condition are very closely related to the geodesic escape. Since the geodesic depends on a nonlinear ODE, they are hard to check. On the other hand, the non-trapping assumption and geometric control condition are not sufficient to derive Morawetz estimates for hyperbolic equations on global space. In comparison to the existing studies, we here take advantage of the metric $g$ to establish Morawetz estimates for the Schrödinger equation.

As is known, the multiplier method is a simple and effective tool to deal with the energy estimate on PDEs. In particular, the celebrated Morawetz multipliers introduced by [45] have been extensively used to study the energy decay of the wave equation with constant coefficients, see [24, 39, 43, 46] and many others. For bounded domains, Yao [56] developed Morawetz multipliers for the wave equation with variable coefficients, which is a powerful tool in the analysis of systems with variable coefficients and has been extended by [16, 57, 59] and many others mentioned in [58. However, how to establish the Morawetz estimates in non (asymptotically) Euclidean spaces is still an open problem. Therefore, one purpose of this paper is to establish Morawetz estimates on non (asymptotically) Euclidean spaces.

The organization of our paper goes as follows. In Section 2, we will state our main results. Then some multiplier identities and key lemmas for problem (1.9) will be presented in Section 3. We will show Morawetz estimates for the nonlinear Schrödinger equation without dissipation in Section 4. Then proofs of stability of the damped nonlinear Schrödinger equation with non-uniform decay rate will be presented in Section 5. We will prove stability of the damped nonlinear Schrödinger equation with uniform decay rate in Section 6. Finally, the proof for Assumption (U1) and Assumption (U2) hereinafter under stronger geometric condition is given in Appendix. 


\section{Main results}

\subsection{Well-posedness}

Denote

$$
\begin{gathered}
C_{1}^{\infty}(\Omega)=\left\{w \in C^{\infty}(\bar{\Omega}) \quad \text { and } \quad \int_{\Omega}|w|^{2} d x_{g}<+\infty\right\} . \\
C_{2}^{\infty}(\Omega)=\left\{w \in C^{\infty}(\bar{\Omega}) \quad \text { and } \quad \int_{\Omega}\left(|w|^{2}+\left|\nabla_{g} w\right|_{g}^{2}\right) d x_{g}<+\infty\right\} . \\
C_{3}^{\infty}(\Omega)=\left\{w \in C^{\infty}(\bar{\Omega}) \quad \text { and } \quad \int_{\Omega}\left(|w|^{2}+\left|\nabla_{g} w\right|_{g}^{2}+\left|\Delta_{g} u\right|^{2}\right) d x_{g}<+\infty\right\} .
\end{gathered}
$$

Let $L^{2}(\Omega)$ be the closure of $C_{1}^{\infty}(\Omega)$ with respect to the tolopogy

$$
\|w(x)\|_{L^{2}(\Omega)}^{2}=\int_{\Omega}|w|^{2} d x_{g}
$$

$H^{1}(\Omega)$ be the closure of $C_{2}^{\infty}(\Omega)$ with respect to the tolopogy

$$
\|w(x)\|_{H^{1}(\Omega)}^{2}=\int_{\Omega}\left(|w|^{2}+\left|\nabla_{g} w\right|_{g}^{2}\right) d x_{g}
$$

and $H^{2}(\Omega)$ be the closure of $C_{3}^{\infty}(\Omega)$ with respect to the tolopogy

$$
\|w(x)\|_{H^{2}(\Omega)}^{2}=\int_{\Omega}\left(|w|^{2}+\left|\nabla_{g} w\right|_{g}^{2}+\left|\Delta_{g} u\right|^{2}\right) d x_{g} .
$$

Denote

$$
H_{\Gamma}^{1}(\Omega)=\left\{w \in H^{1}(\Omega),\left.\quad w\right|_{\Gamma}=0\right\} .
$$

It is well-known that the system (1.9) is subcritical and has been studied extensively in the Euclidean geometry for large classes of nonlinearities, see the books [22, 28, and the references therein. On the hyperbolic spaces, well-posedness and scattering of the system (1.9) without dissipation have been proved in [31, 32]. Therefore, throughout the paper, we assume that the following condition holds true.

Assumption (S) The system (1.9) is well-posed such that

$$
u \in C\left([0,+\infty), H_{\Gamma}^{1}(\Omega) \bigcap H^{2}(\Omega)\right) .
$$

\subsection{Morawetz estimates for the nonlinear Schrödinger equation in non (asymptotically) Euclidean spaces}

The main geometric conditions for Morawetz estimates of the nonlinear Schrödinger equation in non (asymptotically) Euclidean spaces are given by the following assumption.

Assumption (A) Assume that

$$
a(x) \equiv 0 \quad \text { in } \quad \Omega,
$$




$$
\begin{gathered}
G(x) \frac{\partial}{\partial r}=\frac{\partial}{\partial r}, \quad x \in \mathbb{R}^{n} \\
\left\langle\left((1-\alpha(x)) G(x)+\frac{r}{2} \frac{\partial G(x)}{\partial r}\right) X, X\right\rangle \geq 0 \quad \text { for } \quad X \in S(r)_{x}, \quad x \in \Omega, \\
\operatorname{det}(G(x))=c_{0} r^{d}, \quad x \in \Omega
\end{gathered}
$$

where $c_{0}>0, d$ are constants and $\alpha(x)$ is a continuous nonnegative function defined on $\mathbb{R}^{n}$.

Remark 2.1. Let $(r, \theta)=\left(r, \theta_{1}, \theta_{2}, \cdots, \theta_{n-1}\right)$ be the polar coordinates of $x \in \mathbb{R}^{n}$ in the Euclidean metric. From (2.10), we have

$$
g=d r^{2}+\sum_{i, j=1}^{n-1} \gamma_{i j}(r, \theta) d \theta_{i} d \theta_{j}, \quad x \in \mathbb{R}^{n},
$$

which implies $r(x)=|x|$ is the geodesic distance function of $\left(\mathbb{R}^{n}, g\right)$ from $x$ to the origin $O$.

Remark 2.2. Let Assumption (A) hold true. It follows from relations (4.6) and (4.7) hereinafter that

$$
\begin{aligned}
\frac{(n+d / 2-1)}{r} & =\frac{n-1}{r}+\frac{\partial \ln \sqrt{\operatorname{det}(G(x))}}{\partial r}=\Delta_{g} r=t r D^{2} r \\
& \geq(n-1) \frac{\alpha(x)}{r} \geq 0, \quad x \in \Omega .
\end{aligned}
$$

Then

$$
d \geq 2(1-n)
$$

Example 2.1. Let $d_{1}=d_{2}$ and $G(x)$ satisfy

$$
G(x)=\frac{x \otimes x}{|x|^{2}}+f(r)\left(I_{n}-\frac{x \otimes x}{|x|^{2}}\right), \quad x \in \mathbb{R}^{n},
$$

where $f(r)$ is a smooth function defined on $[0,+\infty)$ such that

$$
f(r)=r^{m}, \quad|x| \geq d_{1} \quad \text { and } \quad f(r)=1, \quad|x|<\frac{d_{1}}{2} .
$$

Therefore,

$$
\begin{gathered}
G(x) \frac{\partial}{\partial r}=\frac{\partial}{\partial r}, \quad x \in \mathbb{R}^{n}, \\
\left\langle\left(\frac{1}{2} \frac{\partial G(x)}{\partial r}\right) X, X\right\rangle=\frac{m}{2 r}|X|_{g}^{2} \quad \text { for } \quad X \in S(r)_{x}, \quad|x| \geq d_{1}, \\
\operatorname{det}(G(x))=r^{m(n-1)} \quad \text { for } \quad|x| \geq d_{1} .
\end{gathered}
$$

Let

$$
\alpha(x)=1-\frac{m}{2}, \quad d=m(n-1) .
$$

Then, (2.10), 2.11) and (2.12) hold true. 
Theorem 2.1. Let Assumption (A) hold true. Assume that

$$
\frac{\partial r}{\partial \nu} \leq 0, \quad x \in \Gamma
$$

Then there exists a positive constant $C$ such that for $d=2(3-n)$,

$$
\int_{0}^{T} \int_{\Omega} \frac{|u|^{p+1}}{r} d x_{g} d t+\int_{0}^{T} \int_{\Omega} \frac{\alpha(x)}{r}\left(\left|\nabla_{g} u\right|_{g}^{2}-\left|u_{r}\right|^{2}\right) d x_{g} d t \leq C E(0),
$$

and for $d>2(3-n)$,

$$
\int_{0}^{T} \int_{\Omega}\left(\frac{|u|^{2}}{r^{3}}+\frac{|u|^{p+1}}{r}\right) d x_{g} d t+\int_{0}^{T} \int_{\Omega} \frac{\alpha(x)}{r}\left(\left|\nabla_{g} u\right|_{g}^{2}-\left|u_{r}\right|^{2}\right) d x_{g} d t \leq C E(0) .
$$

\subsection{Stability of the damped nonlinear Schrödinger equation with non- uniform energy decay rate}

The main geometric conditions for stability of the damped nonlinear Schrödinger equation with non-uniform energy decay rate are given by the following assumption.

Assumption (B) There exist constants $R_{0}>d_{2}, 0<\delta \leq 1$ such that

$$
\left\langle\left((1-\delta) G(x)+\frac{r}{2} \frac{\partial G(x)}{\partial r}\right) X, X\right\rangle \geq 0 \quad \text { for } \quad X \in \mathbb{R}_{x}^{n}, \quad x \in \Omega\left(R_{0}\right),
$$

and $a(x)$ satisfies

$$
a(x) \geq a_{0}>0, \quad x \in\left(\Omega \backslash \Omega\left(R_{0}-\varepsilon_{0}\right)\right) \bigcup \Gamma\left(\varepsilon_{1}\right),
$$

for some $0<2 \varepsilon_{1}<\varepsilon_{0}<R_{0}-d_{2}$, where

$$
\Gamma(\varepsilon)=\bigcup_{x \in \Gamma}\{y \in \Omega|| y-x \mid<\varepsilon\}
$$

and for any $\epsilon>0$, there exists $C_{\epsilon}$ such that

$$
\left|\Delta_{g} a(x)\right| \leq C_{\epsilon} a(x)+\epsilon, \quad x \in \Omega .
$$

To prove the stability of the system (1.9), the following assumptions are also considered.

Assumption (U1) Let $\widehat{\Omega} \subset \mathbb{R}^{n}$ be a bounded domain with smooth boundary and $\omega$ be an open subset of $\widehat{\Omega}$ such that

$$
\omega \supset \bigcup_{x \in \partial \widehat{\Omega}}\{y \in \widehat{\Omega}|| y-x \mid<\xi\},
$$

for some $\xi>0$. Assume that $\omega$ satisfies geometric control condition:

(GCC) There exists constant $T_{0}>0$ such that for any $x \in \widehat{\Omega}$ and any unit-speed geodesic $\gamma(t)$ of $\left(\mathbb{R}^{n}, g\right)$ starting at $x$, there exists $t<T_{0}$ such that $\gamma(t) \subset \omega$. 
Then there exists $T_{1} \geq 0$ such that for any $T>T_{1}$, the only solution $u$ in $C\left([0, T], H^{1}(\widehat{\Omega})\right)$ to the system

$$
\left\{\begin{array}{l}
i u_{t}+\Delta_{g} u=0 \quad(x, t) \in \widehat{\Omega} \times(0, T), \\
u=0 \quad(x, t) \in \omega \times(0, T),
\end{array}\right.
$$

is the trivial one $u \equiv 0$.

Assumption (U2) Let $\widehat{\Omega} \subset \mathbb{R}^{n}$ be a bounded domain with smooth boundary and $\omega$ be an open subset of $\widehat{\Omega}$ such that

$$
\omega \supset \bigcup_{x \in \partial \widehat{\Omega}}\{y \in \widehat{\Omega}|| y-x \mid<\xi\},
$$

for some $\xi>0$. Assume that $\omega$ satisfies geometric control condition:

(GCC) There exists constant $T_{0}>0$ such that for any $x \in \widehat{\Omega}$ and any unit-speed geodesic $\gamma(t)$ of $\left(\mathbb{R}^{n}, g\right)$ starting at $x$, there exists $t<T_{0}$ such that $\gamma(t) \in \omega$.

Then there exists $T_{1} \geq 0$ such that for any $T>T_{1}$, the only solution $u$ in $C\left([0, T], H^{1}(\widehat{\Omega})\right)$ to the system

$$
\left\{\begin{array}{l}
i u_{t}+\Delta_{g} u-|u|^{p-1} u=0 \quad(x, t) \in \widehat{\Omega} \times(0, T), \\
u=0 \quad(x, t) \in \omega \times(0, T) .
\end{array}\right.
$$

is the trivial one $u \equiv 0$.

Remark 2.3. If $T_{1}=0$, which implies $T$ can be arbitrary small in (2.30) and (2.32), Assumption (U1) and Assumption (U2) are called as unique continuation condition. On Euclidean space, unique continuation condition for linear(or nonlinear) Schrödinger equation has been proved by [26, 27, 33, 34, 36, 55] and the references therein. On Riemannian manifold, under the assumption that unique continuation condition for linear Schrödinger equation holds true, unique continuation condition for the nonlinear Schrödinger equation was proved by [40] in dimension 3 and [25] in dimension 2 .

By the equivalent relation between the controllability and the observability estimate [42], Assumption (U1) follows from Theorem 4.4 in [41]. However, a detailed proof of Theorem 4.4 in 41 is not provided.

Under a stronger geometric condition than (GCC), we can prove Assumption (U1) and Assumption (U2) directly by multiplier methods. See Proposition (A.1) and Proposition (A.2) in the Appendix.

Theorem 2.2. Let Assumption (B), Assumption (U1) and Assumption (U2) hold true. Assume that $\left\|u_{0}\right\|_{L^{2}(\Omega)} \leq E_{0}$. Then there exist positive constants $C_{1}$ and $C_{2}$, which are dependent on $E_{0}$, such that

$$
E(t) \leq C_{1} e^{-C_{2} t} E(0), \quad \forall t>0
$$




\subsection{Stability of the damped nonlinear Schrödinger equation with uni- form energy decay rate}

The main geometric conditions for stability of the damped nonlinear Schrödinger equation with uniform energy decay rate are given by the following assumption.

Assumption (C) There exist constants $R_{0}>d_{2}, 0<\delta \leq 1$ such that

$$
\begin{gathered}
G(x) \frac{\partial}{\partial r}=\frac{\partial}{\partial r}, \quad|x| \leq R_{0} \quad \text { and } \quad \operatorname{det}(G(x))=c_{0} r^{d}, \quad x \in \Omega\left(R_{0}\right) \\
\left\langle\left((1-\delta) G(x)+\frac{r}{2} \frac{\partial G(x)}{\partial r}\right) X, X\right\rangle \geq 0 \quad \text { for } \quad X \in \mathbb{R}_{x}^{n}, \quad x \in \Omega\left(R_{0}\right)
\end{gathered}
$$

where $c_{0}>0, d$ are constants and $a(x)$ satisfies

$$
a(x) \geq a_{0}>0, \quad x \in \Omega \backslash \Omega\left(R_{0}-\varepsilon_{0}\right),
$$

for some $0<\varepsilon_{0}<R_{0}-d_{2}$ and for any $\epsilon>0$, there exists $C_{\epsilon}$ such that

$$
\left|\Delta_{g} a(x)\right| \leq C_{\epsilon} a(x)+\epsilon, \quad x \in \Omega .
$$

Remark 2.4. Let Assumption (C) hold true. It follows from the relations 4.6) and 4.7) hereinafter that

$$
\begin{aligned}
\frac{(n+d / 2-1)}{r} & =\frac{n-1}{r}+\frac{\partial \ln \sqrt{\operatorname{det}(G(x))}}{\partial r}=\Delta_{g} r=t r D^{2} r \\
& \geq(n-1) \frac{\delta}{r}, \quad x \in \Omega\left(R_{0}\right) .
\end{aligned}
$$

Then

$$
d \geq 2(n-1)(\delta-1)
$$

Theorem 2.3. Let Assumption (C) hold true. Assume that

$$
\frac{\partial r}{\partial \nu} \leq 0, \quad x \in \Gamma .
$$

Then there exist positive constants $C_{1}, C_{2}$ such that

$$
E(t) \leq C_{1} e^{-C_{2} t} E(0), \quad \forall t>0
$$

\section{Multiplier Identities and Key Lemmas}

We need to establish several multiplier identities, which are useful for our problem.

Lemma 3.1. Let $\widehat{\Omega} \subset \mathbb{R}^{n}$ be a bounded domain with smooth boundary. Suppose that $u(x, t)$ solves the following equation:

$$
i u_{t}+\Delta_{g} u+i a(x) u-|u|^{p-1} u=0 \quad(x, t) \in \widehat{\Omega} \times(0,+\infty) .
$$


Let $\mathcal{H}$ be a $C^{1}$ vector field defined on $\overline{\widehat{\Omega}}$. Then

$$
\begin{aligned}
& \int_{0}^{T} \int_{\partial \widehat{\Omega}} \operatorname{Re}\left(\frac{\partial u}{\partial \hat{\nu}} \mathcal{H}(\bar{u})\right) d \Gamma_{g} d t+\frac{1}{2} \int_{0}^{T} \int_{\partial \widehat{\Omega}}\left(\operatorname{Im}\left(u \bar{u}_{t}\right)-\left|\nabla_{g} u\right|_{g}^{2}-\frac{2}{p+1}|u|^{p+1}\right)\langle\mathcal{H}, \hat{\nu}\rangle_{g} d \Gamma_{g} d t \\
= & \left.\frac{1}{2} \int_{\widehat{\Omega}} \operatorname{Im}(u \mathcal{H}(\bar{u})) d x_{g}\right|_{0} ^{T}+\int_{0}^{T} \int_{\widehat{\Omega}} \operatorname{Re} D \mathcal{H}\left(\nabla_{g} \bar{u}, \nabla_{g} u\right) d x_{g} d t \\
& +\int_{0}^{T} \int_{\widehat{\Omega}} \operatorname{Im}(a(x) u \mathcal{H}(\bar{u})) d x_{g} d t \\
& +\frac{1}{2} \int_{0}^{T} \int_{\widehat{\Omega}}\left(\operatorname{Im}\left(u \bar{u}_{t}\right)-\left|\nabla_{g} u\right|_{g}^{2}-\frac{2}{p+1}|u|^{p+1}\right) \operatorname{div}_{g} \mathcal{H} d x_{g} d t
\end{aligned}
$$

where $\hat{\nu}(x)$ is the unit normal vector outside $\widehat{\Omega}$ in $\left(\mathbb{R}^{n}, g\right)$ for $x \in \partial \widehat{\Omega}$ and

$$
d \Gamma_{g}=\sqrt{\operatorname{det}(G(x))} d \Gamma .
$$

Moreover, assume that the real function $P \in C^{2}(\overline{\widehat{\Omega}})$. Then

$$
\begin{aligned}
& \int_{0}^{T} \int_{\widehat{\Omega}}\left(\operatorname{Im}\left(u \bar{u}_{t}\right)-\left|\nabla_{g} u\right|_{g}^{2}-|u|^{p+1}\right) P d x_{g} d t \\
& =\frac{1}{2} \int_{0}^{T} \int_{\partial \widehat{\Omega}}|u|^{2} \frac{\partial P}{\partial \hat{\nu}} d \Gamma_{g} d t-\frac{1}{2} \int_{0}^{T} \int_{\widehat{\Omega}}|u|^{2}\left(\Delta_{g} P\right) d x_{g} d t \\
& \quad-\int_{0}^{T} \int_{\partial \widehat{\Omega}} \operatorname{Re}\left(P \bar{u} \frac{\partial u}{\partial \hat{\nu}}\right) d \Gamma_{g} d t .
\end{aligned}
$$

Proof. Firstly, we multiply (3.1) by $\mathcal{H}(\bar{u})$ and integrate over $\widehat{\Omega} \times(0, T)$. We deduce that

$$
\begin{aligned}
\operatorname{Re}\left(i u_{t} \mathcal{H}(\bar{u})\right) & =-\operatorname{Im}\left(u_{t} \mathcal{H}(\bar{u})\right) \\
& =-\frac{1}{2} \operatorname{Im}\left(u_{t} \mathcal{H}(\bar{u})-\bar{u}_{t} \mathcal{H}(u)\right) \\
& =-\frac{1}{2} \operatorname{Im}\left((u \mathcal{H}(\bar{u}))_{t}-\mathcal{H}\left(u \bar{u}_{t}\right)\right) \\
& =-\frac{1}{2} \operatorname{Im}(u \mathcal{H}(\bar{u}))_{t}+\frac{1}{2} \operatorname{Im} \mathcal{H}\left(u \bar{u}_{t}\right) \\
& =-\frac{1}{2} \operatorname{Im}(u \mathcal{H}(\bar{u}))_{t}+\frac{1}{2} \operatorname{Im} \operatorname{div}_{g}\left(u \bar{u}_{t} \mathcal{H}\right)-\frac{1}{2} \operatorname{Im}\left(u \bar{u}_{t} \operatorname{div}_{g} \mathcal{H}\right)
\end{aligned}
$$

$\left.\operatorname{Re}\left(\mathcal{H}(\bar{u}) \Delta_{g} u\right)\right)=\operatorname{Re}\left(\operatorname{div}_{g} \mathcal{H}(\bar{u}) \nabla_{g} u-\nabla_{g} u\left\langle\mathcal{H}, \nabla_{g} \bar{u}\right\rangle_{g}\right)$

$$
\begin{aligned}
= & \operatorname{Re} \operatorname{div}_{g} \mathcal{H}(\bar{u}) \nabla_{g} u-\operatorname{Re} \nabla_{g} u\left\langle\mathcal{H}, \nabla_{g} \bar{u}\right\rangle_{g} \\
= & \operatorname{Re} \operatorname{div}_{g} \mathcal{H}(\bar{u}) \nabla_{g} u-\operatorname{Re} D \mathcal{H}\left(\nabla_{g} \bar{u}, \nabla_{g} u\right)-\operatorname{Re} D^{2} \bar{u}\left(\mathcal{H}, \nabla_{g} u\right) \\
= & \operatorname{Re} \operatorname{div}_{g} \mathcal{H}(\bar{u}) \nabla_{g} u-\operatorname{Re} D \mathcal{H}\left(\nabla_{g} \bar{u}, \nabla_{g} u\right)-\operatorname{Re} D^{2} \bar{u}\left(\nabla_{g} u, \mathcal{H}\right) \\
= & \operatorname{Re} \operatorname{div}_{g} \mathcal{H}(\bar{u}) \nabla_{g} u-\operatorname{Re} D \mathcal{H}\left(\nabla_{g} \bar{u}, \nabla_{g} u\right)-\frac{1}{2} \mathcal{H}\left(\left|\nabla_{g} u\right|_{g}^{2}\right) \\
= & \operatorname{Re} \operatorname{div}_{g} \mathcal{H}(\bar{u}) \nabla_{g} u-\operatorname{Re} D \mathcal{H}\left(\nabla_{g} \bar{u}, \nabla_{g} u\right)-\frac{1}{2} \operatorname{div}_{g}\left(\left|\nabla_{g} u\right|_{g}^{2} \mathcal{H}\right) \\
& \quad+\frac{1}{2}\left|\nabla_{g} u\right|_{g}^{2} \operatorname{div}_{g} \mathcal{H},
\end{aligned}
$$


and

$$
\begin{aligned}
\operatorname{Re}\left(i a(x) u-|u|^{p-1} u\right) \mathcal{H}(\bar{u})= & -\operatorname{Im}(a(x) u \mathcal{H}(\bar{u})) \\
& -\frac{1}{p+1} \operatorname{div}_{g}\left(|u|^{p+1} \mathcal{H}\right)+\frac{|u|^{p+1}}{p+1} \operatorname{div}_{g} \mathcal{H} .
\end{aligned}
$$

The equality (3.2) follows from Green's formula.

In addition, by multiplying (3.1) by $P \bar{u}$ and integrating over $\widehat{\Omega} \times(0, T)$, we obtain

$$
\begin{aligned}
& \operatorname{Re}\left(i P u_{t} \bar{u}\right)=-\operatorname{Im}\left(P u_{t} \bar{u}\right)=\operatorname{Im}\left(P u \bar{u}_{t}\right), \\
\operatorname{Re}\left(P \bar{u} \Delta_{g} u\right)= & \operatorname{Re}\left(\operatorname{div}_{g} P \bar{u} \nabla_{g} u-\nabla_{g} u(P \bar{u})\right) \\
= & \operatorname{Re} \operatorname{div}_{g} P \bar{u} \nabla_{g} u-P\left|\nabla_{g} u\right|_{g}^{2}-\frac{1}{2} \nabla_{g} P\left(|u|^{2}\right) \\
= & \operatorname{Re} \operatorname{div}_{g} P \bar{u} \nabla_{g} u-P\left|\nabla_{g} u\right|_{g}^{2}-\frac{1}{2} \operatorname{div}_{g}|u|^{2} \nabla_{g} P+\frac{1}{2}|u|^{2} \Delta_{g} P,
\end{aligned}
$$

and

$$
\begin{aligned}
\operatorname{Re}\left(i a(x) u-|u|^{p-1} u\right) P \bar{u} & =\operatorname{Re}\left(i a(x) P|u|^{2}\right)-P|u|^{p+1} \\
& =-P|u|^{p+1} .
\end{aligned}
$$

The equality (3.4) follows from Green's formula.

The following lemma will be utilized frequently in our subsequent proof.

Lemma 3.2. Let $x_{0} \in \mathbb{R}^{n}$ be a fixed point. Let $H(x)=x-x_{0}$, then

$$
D H(X, X)=\left\langle\left(G(x)+\frac{\widehat{r}(x)}{2} \frac{\partial G(x)}{\partial \widehat{r}}\right) X, X\right\rangle, \quad \text { for } X \in \mathbb{R}_{x}^{n}, x \in \mathbb{R}^{n},
$$

where $\widehat{r}(x)=\left|x-x_{0}\right|$.

Proof. Let $x \in \mathbb{R}^{n}, X=\sum_{i=1}^{n} X_{i} \frac{\partial}{\partial x_{i}} \in \mathbb{R}_{x}^{n}$. Note that

$$
H(x)=\sum_{i=1}^{n}\left(x_{i}-x_{0, i}\right) \frac{\partial}{\partial x_{i}} .
$$

Then, we deduce that

$$
\begin{aligned}
D H(X, X) & =\sum_{i, j, k=1}^{n}\left\langle D_{\frac{\partial}{\partial x_{i}}}\left(\left(x_{k}-x_{0, k}\right) \frac{\partial}{\partial x_{k}}\right), \frac{\partial}{\partial x_{j}}\right\rangle_{g} X_{i} X_{j} \\
& =\sum_{i, j=1}^{n} g_{i j} X_{i} X_{j}+\sum_{i, j, k=1}^{n}\left(x_{k}-x_{0, k}\right)\left\langle D_{\frac{\partial}{\partial x_{i}}} \frac{\partial}{\partial x_{k}}, \frac{\partial}{\partial x_{j}}\right\rangle_{g} X_{i} X_{j} \\
& =|X|_{g}^{2}+\sum_{i, j, k=1}^{n}\left(x_{k}-x_{0, k}\right)\left\langle D_{\frac{\partial}{\partial x_{k}}} \frac{\partial}{\partial x_{i}}, \frac{\partial}{\partial x_{j}}\right\rangle_{g} X_{i} X_{j} \\
& =|X|_{g}^{2}+\sum_{i, j, k=1}^{n} \frac{\left(x_{k}-x_{0, k}\right)}{2} \frac{\partial g_{i j}}{\partial x_{k}} X_{i} X_{j} \\
& =\left\langle\left(G(x)+\frac{\widehat{r}(x)}{2} \frac{\partial G(x)}{\partial \widehat{r}}\right) X, X\right\rangle
\end{aligned}
$$


The following lemmas show the relationship between the metric $g$ and geometric control condition.

Lemma 3.3. Let $\widehat{\Omega} \subset \mathbb{R}^{n}$ be a bounded domain and $x_{0} \in \mathbb{R}^{n}$ be a fixed point. Assume that there exists $\delta>0$ such that

$$
\left\langle\left((1-\delta) G(x)+\frac{\widehat{r}(x)}{2} \frac{\partial G(x)}{\partial \widehat{r}}\right) X, X\right\rangle \geq 0, \quad \text { for } \quad X \in \mathbb{R}_{x}^{n}, x \in \overline{\widehat{\Omega}}
$$

where $\widehat{r}(x)=\left|x-x_{0}\right|$. Then, for any $x \in \widehat{\Omega}$ and any unit-speed geodesic $\gamma(t)$ starting at $x$, if

$$
\gamma(t) \in \widehat{\Omega}, \quad 0 \leq t \leq t_{0}
$$

then

$$
t_{0} \leq \frac{2}{\delta} \sup \left\{\left|x-x_{0}\right| g(x) \mid x \in \overline{\widehat{\Omega}}\right\}
$$

Proof. Let $H(x)=x-x_{0}$. It follows from (3.11) that

$$
D H(X, X) \geq \delta|X|_{g}^{2} \quad \text { for all } X \in \mathbb{R}_{x}^{n}, \quad x \in \overline{\widehat{\Omega}} .
$$

Note that

$$
\left|\gamma^{\prime}(t)\right|_{g}=1, \quad D_{\gamma^{\prime}(t)} \gamma^{\prime}(t)=0
$$

Then

$$
\left.\left\langle H, \gamma^{\prime}(t)\right\rangle_{g}\right|_{0} ^{t_{0}}=\int_{0}^{t_{0}} \gamma^{\prime}(t)\left\langle H, \gamma^{\prime}(t)\right\rangle_{g} d t=\int_{0}^{t_{0}} D H\left(\gamma^{\prime}(t), \gamma^{\prime}(t)\right) d t \geq \delta t_{0}
$$

Hence

$$
t_{0} \leq \frac{2}{\delta} \sup \left\{|H|_{g}(x) \mid x \in \overline{\widehat{\Omega}}\right\}
$$

Lemma 3.4. Assume that

$$
\begin{gathered}
G(x) \frac{\partial}{\partial r}=\frac{\partial}{\partial r}, \quad x \in \mathbb{R}^{n}, \\
\frac{\partial G(x)}{\partial r}=-\frac{2}{r_{2}} G(x)\left(I_{n}-\frac{x \otimes x}{|x|^{2}}\right), \quad|x|=r_{2},
\end{gathered}
$$

where $r_{2}$ is a positive constant. Then, for any $x \in S\left(r_{2}\right)$ and any unit-speed geodesic $\gamma(t)$ starting at $x$ with

$$
\gamma^{\prime}(0) \in S\left(r_{2}\right)_{x},
$$

we have

$$
\gamma(t) \in S\left(r_{2}\right), \quad \forall t \geq 0
$$


Proof Note that

$$
D(r D r)=D r \otimes D r+r D^{2} r .
$$

With (3.11), we obtain

$$
D^{2} r(X, X)=\left\langle\left(\frac{1}{r} G(x)+\frac{1}{2} \frac{\partial G(x)}{\partial r}\right) X, X\right\rangle=0 \quad \text { for } \quad X \in S\left(r_{2}\right)_{x}, \quad x \in S\left(r_{2}\right) .
$$

Let $\widehat{g}$ be a Riemannian metric induced by $g$ in $S\left(r_{2}\right)$ and $\widehat{D}$ be the associated LeviCivita connection.

Let $\widehat{\gamma}(t)$ be a unit-speed geodesic of $\left(S\left(r_{2}\right), \widehat{g}\right)$ starting at $x \in S\left(r_{2}\right)$, then

$$
\left\langle\widehat{\gamma}^{\prime}(t), \frac{\partial}{\partial r}\right\rangle_{g}=0, \quad \widehat{D}_{\widehat{\gamma}^{\prime}(t)} \widehat{\gamma}^{\prime}(t)=0, \quad \forall t \geq 0
$$

Therefore,

$$
\begin{aligned}
D_{\widehat{\gamma}^{\prime}(t)} \widehat{\gamma}^{\prime}(t) & =\widehat{D}_{\widehat{\gamma}^{\prime}(t)} \widehat{\gamma}^{\prime}(t)+\left\langle D_{\widehat{\gamma}^{\prime}(t)} \widehat{\gamma}^{\prime}(t), \frac{\partial}{\partial r}\right\rangle_{g} \frac{\partial}{\partial r} \\
& =\widehat{D}_{\widehat{\gamma}^{\prime}(t)} \widehat{\gamma}^{\prime}(t)-D^{2} r\left(\widehat{\gamma}^{\prime}(t), \widehat{\gamma}^{\prime}(t)\right) \frac{\partial}{\partial r}=0,
\end{aligned}
$$

which implies $\widehat{\gamma}(t)$ is also a geodesic of $\left(\mathbb{R}^{n}, g\right)$.

\section{Proofs of Morawetz estimates in non (asymptotically) Eu- clidean spaces}

Lemma 4.1. Let $u(x, t)$ solve the system (1.9). Then

$$
\begin{aligned}
\left.\int_{\Omega}|u|^{2} d x_{g}\right|_{0} ^{T}= & -2 \int_{0}^{T} \int_{\Omega} a(x)|u|^{2} d x_{g} d t \\
\left.\int_{\Omega}\left(\left|\nabla_{g} u\right|_{g}^{2}+\frac{2}{p+1}|u|^{p+1}\right) d x_{g}\right|_{0} ^{T} & =-2 \int_{0}^{T} \int_{\Omega} a(x)\left(\left|\nabla_{g} u\right|_{g}^{2}+|u|^{p+1}\right) d x_{g} d t \\
& +\int_{0}^{T} \int_{\Omega}|u|^{2}\left(\Delta_{g} a(x)\right) d x_{g} d t
\end{aligned}
$$

for any $T>0$.

Proof. Multiplying the Schrödinger equation in (1.9) by $2 \bar{u}$ and integrating over $\Omega \times(0, T)$, we have

$$
\left.\int_{\Omega}|u|^{2} d x_{g}\right|_{0} ^{T}=-2 \int_{0}^{T} \int_{\Omega} a(x)|u|^{2} d x_{g}
$$

After multiplying the Schrödinger equation in (1.9) by $2 \bar{u}_{t}$ and then integrating over $\Omega \times(0, T)$, we obtain

$$
\left.\int_{\Omega}\left(\left|\nabla_{g} u\right|_{g}^{2}+\frac{2}{p+1}|u|^{p+1}\right) d x_{g}\right|_{0} ^{T}=-2 \int_{0}^{T} \int_{\Omega} \operatorname{Im}\left(a(x) u \bar{u}_{t}\right) d x_{g} d t .
$$


Let $P=a(x)$ and $\widehat{\Omega}=\Omega(a)$ in (3.4). Substituting (3.4) into (4.4), letting $a \rightarrow+\infty$, we get

$$
\begin{aligned}
\left.\int_{\Omega}\left(\left|\nabla_{g} u\right|_{g}^{2}+\frac{2}{p+1}|u|^{p+1}\right) d x_{g}\right|_{0} ^{T} & =-2 \int_{0}^{T} \int_{\Omega} a(x)\left(\left|\nabla_{g} u\right|_{g}^{2}+|u|^{p+1}\right) d x_{g} d t \\
& +\int_{0}^{T} \int_{\Omega}|u|^{2}\left(\Delta_{g} a(x)\right) d x_{g} d t
\end{aligned}
$$

Lemma 4.2. Let Assumption (A) hold true. Then

$$
\begin{gathered}
D^{2} r(X, X) \geq \frac{\alpha(x)}{r}|X|_{g}^{2} \quad \text { for } \quad \text { all } X \in S(r)_{x}, x \in \Omega, \\
\Delta_{g} r=\frac{n+d / 2-1}{r} \quad \text { for } x \in \Omega .
\end{gathered}
$$

Proof. Note that

$$
D(r D r)=D r \otimes D r+r D^{2} r
$$

With (3.11), we obtain

$$
D^{2} r(X, X)=\left\langle\left(\frac{1}{r} G(x)+\frac{1}{2} \frac{\partial G(x)}{\partial r}\right) X, X\right\rangle \geq \frac{\alpha(x)}{r}|X|_{g}^{2} \quad \text { for } \quad X \in S(r)_{x}, \quad x \in \Omega,
$$

and

$$
\Delta_{g} r=\frac{n-1}{r}+\frac{\partial \ln \sqrt{\operatorname{det}(G(x))}}{\partial r}=\frac{n+d / 2-1}{r} \quad \text { for } x \in \Omega
$$

\section{Proof of Theorem 2.1}

Let $\mathcal{H}=\frac{\partial}{\partial r}$ and $\widehat{\Omega}=\Omega(h)$ in (3.2). It follows from (3.2), (4.6) and (4.7), that

$$
\begin{aligned}
\int_{0}^{T} & \int_{\partial \Omega(h)} \operatorname{Re}\left(\frac{\partial u}{\partial \hat{\nu}} \mathcal{H}(\bar{u})\right) d \Gamma_{g} d t \\
& +\frac{1}{2} \int_{0}^{T} \int_{\partial \Omega(h)}\left(\operatorname{Im}\left(u \bar{u}_{t}\right)-\left|\nabla_{g} u\right|_{g}^{2}-\frac{2}{p+1}|u|^{p+1}\right)\langle\mathcal{H}, \hat{\nu}\rangle_{g} d \Gamma_{g} d t \\
\geq & \left.\frac{1}{2} \int_{\Omega(h)} \operatorname{Im}\left(u \bar{u}_{r}\right) d x_{g}\right|_{0} ^{T}+\int_{0}^{T} \int_{\Omega(h)} \frac{\alpha(x)}{r}\left(\left|\nabla_{g} u\right|_{g}^{2}-\left|u_{r}\right|^{2}\right) d x_{g} d t \\
& +\int_{0}^{T} \int_{\Omega(h)}\left(\operatorname{Im}\left(u \bar{u}_{t}\right)-\left|\nabla_{g} u\right|_{g}^{2}-\frac{2}{p+1}|u|^{p+1}\right) \frac{n-1}{2 r} d x_{g} d t \\
= & \left.\frac{1}{2} \int_{\Omega(h)} \operatorname{Im}\left(u \bar{u}_{r}\right) d x_{g}\right|_{0} ^{T}+\int_{0}^{T} \int_{\Omega(h)} \frac{\alpha(x)}{r}\left(\left|\nabla_{g} u\right|_{g}^{2}-\left|u_{r}\right|^{2}\right) d x_{g} d t \\
& +\int_{0}^{T} \int_{\Omega(h)}\left(\operatorname{Im}\left(u \bar{u}_{t}\right)-\left|\nabla_{g} u\right|_{g}^{2}-|u|^{p+1}\right) \frac{n-1}{2 r} d x_{g} d t \\
& +\int_{0}^{T} \int_{\Omega(h)} \frac{(n-1)(p-1)}{2 r(p+1)}|u|^{p+1} d x_{g} d t .
\end{aligned}
$$


Let $P=\frac{n-1}{2 r}$ and $\widehat{\Omega}=\Omega(h)$ in (3.4). Substituting (3.4) into (4.11), letting $h \rightarrow+\infty$, we obtain

$$
\begin{aligned}
\frac{1}{2} \int_{\Omega} & \left.\operatorname{Im}\left(u \bar{u}_{r}\right) d x_{g}\right|_{0} ^{T}-\frac{n-1}{4} \int_{0}^{T} \int_{\Omega}|u|^{2} \Delta_{g}\left(\frac{1}{r}\right) d x_{g} d t \\
& +\int_{0}^{T} \int_{\Omega} \frac{(p-1)(n-1)}{2(p+1) r}|u|^{p+1} d x_{g} d t+\int_{0}^{T} \int_{\Omega} \frac{\alpha(x)}{r}\left(\left|\nabla_{g} u\right|_{g}^{2}-\left|u_{r}\right|^{2}\right) d x_{g} d t \\
\leq & \Pi_{\Gamma},
\end{aligned}
$$

where

$$
\begin{aligned}
\Pi_{\Gamma}= & \int_{0}^{T} \int_{\Gamma} \operatorname{Re}\left(\frac{\partial u}{\partial \nu} \mathcal{H}(\bar{u})\right) d \Gamma_{g} d t \\
& +\frac{1}{2} \int_{0}^{T} \int_{\Gamma}\left(\operatorname{Im}\left(u \bar{u}_{t}\right)-\left|\nabla_{g} u\right|_{g}^{2}-\frac{2}{p+1}|u|^{p+1}\right)\langle\mathcal{H}, \nu\rangle_{g} d \Gamma_{g} d t \\
& -\frac{1}{2} \int_{0}^{T} \int_{\Gamma}|u|^{2} \frac{\partial P}{\partial \nu} d \Gamma_{g} d t+\int_{0}^{T} \int_{\Gamma} \operatorname{Re}\left(P \bar{u} \frac{\partial u}{\partial \nu}\right) d \Gamma_{g} d t .
\end{aligned}
$$

Since $\left.u\right|_{\Gamma}=0$, we obtain $\left.\nabla_{\Gamma_{g}} \bar{u}\right|_{\Gamma}=0$, that is,

$$
\nabla_{g} \bar{u}=\frac{\partial \bar{u}}{\partial \nu} \nu \quad \text { for } \quad x \in \Gamma .
$$

Similarly, we have

$$
\mathcal{H}(\bar{u})=\left\langle\mathcal{H}, \nabla_{g} \bar{u}\right\rangle_{g}=\frac{\partial \bar{u}}{\partial \nu}\langle\mathcal{H}, \nu\rangle_{g}=\frac{\partial \bar{u}}{\partial \nu} \frac{\partial r}{\partial \nu} \quad \text { for } \quad x \in \Gamma .
$$

Using the formulas (4.14) and (4.15) in the formula (4.13) on the portion $\Gamma$, with (2.22), we obtain

$$
\Pi_{\Gamma}=\frac{1}{2} \int_{0}^{T} \int_{\Gamma}\left|\frac{\partial u}{\partial \nu}\right|^{2} \frac{\partial r}{\partial \nu} d \Gamma_{g} d t \leq 0 .
$$

Substituting (4.16) into (4.12), we have

$$
\begin{aligned}
\frac{1}{2} \int_{\Omega} & \left.\operatorname{Im}\left(u \bar{u}_{r}\right) d x_{g}\right|_{0} ^{T}-\frac{n-1}{4} \int_{0}^{T} \int_{\Omega}|u|^{2} \Delta_{g}\left(\frac{1}{r}\right) d x_{g} d t \\
& +\int_{0}^{T} \int_{\Omega} \frac{(p-1)(n-1)}{2(p+1) r}|u|^{p+1} d x_{g} d t+\int_{0}^{T} \int_{\Omega} \frac{\alpha(x)}{r}\left(\left|\nabla_{g} u\right|_{g}^{2}-\left|u_{r}\right|^{2}\right) d x_{g} d t \\
\leq & 0 .
\end{aligned}
$$

Note that

$$
\Delta_{g}\left(\frac{1}{r}\right)=-\frac{n+d / 2-3}{r} .
$$

With (4.17), we obtain for $d=2(3-n)$,

$$
\begin{aligned}
\frac{1}{2} \int_{\Omega} & \left.\operatorname{Im}\left(u \bar{u}_{r}\right) d x_{g}\right|_{0} ^{T}+\int_{0}^{T} \int_{\Omega} \frac{(p-1)(n-1)}{2(p+1) r}|u|^{p+1} d x_{g} d t \\
& +\int_{0}^{T} \int_{\Omega} \frac{\alpha(x)}{r}\left(\left|\nabla_{g} u\right|_{g}^{2}-\left|u_{r}\right|^{2}\right) d x_{g} d t \\
\leq & 0,
\end{aligned}
$$


and for $d>2(3-n)$,

$$
\begin{aligned}
\frac{1}{2} \int_{\Omega} & \left.\operatorname{Im}\left(u \bar{u}_{r}\right) d x_{g}\right|_{0} ^{T}+\int_{0}^{T} \int_{\Omega} \frac{(n-1)(n+d / 2-3)}{4 r^{3}} u^{2} d x d t \\
& +\int_{0}^{T} \int_{\Omega} \frac{(p-1)(n-1)}{2(p+1) r}|u|^{p+1} d x_{g} d t+\int_{0}^{T} \int_{\Omega} \frac{\alpha(x)}{r}\left(\left|\nabla_{g} u\right|_{g}^{2}-\left|u_{r}\right|^{2}\right) d x d t \\
\leq & 0 .
\end{aligned}
$$

It follows from (2.9), (4.1) and (4.2) that

$$
E(t)=E(0), \quad t>0
$$

The estimates (2.23) and (2.24) follows from (4.19) and (4.20).

\section{Proofs of stability with non-uniform energy decay rate}

From Lemma 3.3, the following lemma holds true.

Lemma 5.1. Let assumption (B) hold true. Then, there exists $t_{0}>0$, for any $x \in$ $\Omega\left(R_{0}\right)$ and any unit-speed geodesic $\gamma(t)$ starting at $x$, there exists $t<t_{0}$ such that

$$
\gamma(t) \in \partial \Omega\left(R_{0}\right)
$$

Lemma 5.2. Let assumption (B) hold true. Let $u(x, t)$ solve the system (1.9). Then

$$
\begin{aligned}
E(0)+\int_{0}^{T} E(t) d t \leq & C \int_{0}^{T} \int_{\Omega} a(x)\left(|u|^{2}+\left|\nabla_{g} u\right|_{g}^{2}+|u|^{p+1}\right) d x_{g} d t \\
& +C \int_{0}^{T} \int_{\Omega\left(R_{0}-\varepsilon_{0}\right)}|u|^{2} d x_{g} d t
\end{aligned}
$$

for sufficiently large $T$.

Proof. Let $b(x) \in C^{\infty}\left(\mathbb{R}^{n}\right)$ be a nonnegative function satisfying

$$
b(x)=1, \quad x \in \Omega\left(R_{0}-\varepsilon_{0}\right) \backslash \Gamma\left(\varepsilon_{1}\right) \quad \text { and } \quad b(x)=0, \quad x \in \mathbb{R}^{n} \backslash \Omega\left(R_{0}\right) .
$$

Let

$$
H(x)=b(x) x, \quad x \in \mathbb{R}^{n} .
$$

It follows from (2.25) and (3.11) that

$$
\begin{gathered}
D H(X, X) \geq \delta|X|_{g}^{2} \quad \text { for } \text { all } X \in \mathbb{R}_{x}^{n}, x \in \Omega\left(R_{0}-\varepsilon_{0}\right) \backslash \Gamma\left(\varepsilon_{1}\right), \\
\operatorname{div}_{g} H=\operatorname{tr} D H \geq n \delta \quad \text { for all } x \in \Omega\left(R_{0}-\varepsilon_{0}\right) \backslash \Gamma\left(\varepsilon_{1}\right) .
\end{gathered}
$$


Let $\mathcal{H}=H$ and $\widehat{\Omega}=\Omega\left(R_{0}\right)$ in (3.2). From (3.2), we have

$$
\begin{aligned}
0 \geq \frac{1}{2} & \left.\int_{\Omega\left(R_{0}\right)} \operatorname{Im}(u H(\bar{u})) d x_{g}\right|_{0} ^{T}+\delta \int_{0}^{T} \int_{\Omega\left(R_{0}-\varepsilon_{0}\right) \backslash \Gamma\left(\varepsilon_{1}\right)}\left|\nabla_{g} u\right|_{g}^{2} d x_{g} d t \\
& -C \int_{0}^{T} \int_{\left(\Omega\left(R_{0}\right) \backslash \Omega\left(R_{0}-\varepsilon_{0}\right)\right) \cup \Gamma\left(\varepsilon_{1}\right)}\left|\nabla_{g} u\right|_{g}^{2} d x_{g} d t+\int_{0}^{T} \int_{\Omega\left(R_{0}\right)} \operatorname{Im}(a(x) u H(\bar{u})) d x_{g} d t \\
& +\frac{1}{2} \int_{0}^{T} \int_{\Omega\left(R_{0}\right)}\left(\operatorname{Im}\left(u \bar{u}_{t}\right)-\left|\nabla_{g} u\right|_{g}^{2}-\frac{2}{p+1}|u|^{p+1}\right) \operatorname{div}{ }_{g} H d x_{g} d t \\
= & \left.\frac{1}{2} \int_{\Omega\left(R_{0}\right)} \operatorname{Im}(u H(\bar{u})) d x_{g}\right|_{0} ^{T}+\delta \int_{0}^{T} \int_{\Omega\left(R_{0}-\varepsilon_{0}\right) \backslash \Gamma\left(\varepsilon_{1}\right)}\left|\nabla_{g} u\right|_{g}^{2} d x_{g} d t \\
& -C \int_{0}^{T} \int_{\left(\Omega\left(R_{0}\right) \backslash \Omega\left(R_{0}-\varepsilon_{0}\right)\right) \cup \Gamma\left(\varepsilon_{1}\right)}\left|\nabla_{g} u\right|_{g}^{2} d x_{g} d t+\int_{0}^{T} \int_{\Omega\left(R_{0}\right)} \operatorname{Im}(a(x) u H(\bar{u})) d x_{g} d t \\
& +\frac{1}{2} \int_{0}^{T} \int_{\Omega\left(R_{0}\right)}\left(\operatorname{Im}\left(u \bar{u}_{t}\right)-\left|\nabla_{g} u\right|_{g}^{2}-|u|^{p+1}\right) \operatorname{div}_{g} H d x_{g} d t \\
& +\int_{0}^{T} \int_{\Omega\left(R_{0}\right)} \frac{(p-1) \operatorname{div}{ }_{g} H}{2(p+1)}|u|^{p+1} d x_{g} d t .
\end{aligned}
$$

Let $P=\frac{\operatorname{div}_{g} H}{2}$ and $\widehat{\Omega}=\Omega\left(R_{0}\right)$ in (3.4). Substituting (3.4) into (5.7), we obtain

$$
\begin{aligned}
& \left.\frac{1}{2} \int_{\Omega\left(R_{0}\right)} \operatorname{Im}(u H(\bar{u})) d x_{g}\right|_{0} ^{T}-\frac{1}{4} \int_{0}^{T} \int_{\Omega\left(R_{0}\right)}|u|^{2} \Delta_{g}\left(\operatorname{div}_{g} H\right) d x_{g} d t \\
& \quad+\int_{0}^{T} \int_{\Omega\left(R_{0}\right)} \operatorname{Im}(a(x) u H(\bar{u})) d x_{g} d t \\
& \quad+\delta \int_{0}^{T} \int_{\Omega\left(R_{0}-\varepsilon_{0}\right) \backslash \Gamma\left(\varepsilon_{1}\right)}\left|\nabla_{g} u\right|_{g}^{2} d x_{g} d t+\int_{0}^{T} \int_{\Omega\left(R_{0}-\varepsilon_{0}\right) \backslash \Gamma\left(\varepsilon_{1}\right)} \frac{n \delta(p-1)}{2(p+1)}|u|^{p+1} d x_{g} d t \\
& \leq C \int_{0}^{T} \int_{\left(\Omega\left(R_{0}\right) \backslash \Omega\left(R_{0}-\varepsilon_{0}\right)\right) \cup \Gamma\left(\varepsilon_{1}\right)}\left(\left|\nabla_{g} u\right|_{g}^{2}+|u|^{p+1}\right) d x_{g} d t .
\end{aligned}
$$

Therefore

$$
\begin{aligned}
& \int_{0}^{T} \int_{\Omega\left(R_{0}-\varepsilon_{0}\right) \backslash \Gamma\left(\varepsilon_{1}\right)}\left(\left|\nabla_{g} u\right|_{g}^{2}+|u|^{p+1}\right) d x_{g} d t \\
\leq & C(E(0)+E(T))+C \int_{0}^{T} \int_{\Omega\left(R_{0}\right)} a(x)\left(|u|^{2}+\left|\nabla_{g} u\right|_{g}^{2}+|u|^{p+1}\right) d x_{g} d t \\
& +C \int_{0}^{T} \int_{\Omega\left(R_{0}-\varepsilon_{0}\right)}|u|^{2} d x_{g} d t .
\end{aligned}
$$

Hence

$$
\begin{aligned}
\int_{0}^{T} E(t) d t \leq & C(E(0)+E(T)) \\
& +C \int_{0}^{T} \int_{\Omega} a(x)\left(|u|^{2}+\left|\nabla_{g} u\right|_{g}^{2}+|u|^{p+1}\right) d x_{g} d t \\
& +C \int_{0}^{T} \int_{\Omega\left(R_{0}-\varepsilon_{0}\right)}|u|^{2} d x_{g} d t
\end{aligned}
$$


With (4.1) and (4.2), we deduce that

$$
\begin{aligned}
C E(T) \leq & C E(0)+C \int_{0}^{T} \int_{\Omega} a(x)\left(|u|^{2}+\left|\nabla_{g} u\right|_{g}^{2}+|u|^{p+1}\right) d x_{g} d t \\
& +\frac{C}{2} \int_{0}^{T} \int_{\Omega}|u|^{2}\left|\Delta_{g} a(x)\right| d x_{g} d t,
\end{aligned}
$$

and

$$
\begin{aligned}
4 C E(0)= & \int_{0}^{4 C} E(t) d t-\int_{0}^{4 C}(E(t)-E(0)) d t \\
\leq & \int_{0}^{4 C} E(t) d t+4 C \int_{0}^{4 C} \int_{\Omega} a(x)\left(|u|^{2}+\left|\nabla_{g} u\right|_{g}^{2}+|u|^{p+1}\right) d x_{g} d t \\
& +2 C \int_{0}^{T} \int_{\Omega}|u|^{2}\left|\Delta_{g} a(x)\right| d x_{g} d t .
\end{aligned}
$$

Substituting (5.11) and (5.12) into (5.10), for $T>4 C$, with (2.28), we have

$$
\begin{aligned}
E(0)+\int_{0}^{T} E(t) d t \leq C & \int_{0}^{T} \int_{\Omega} a(x)\left(|u|^{2}+\left|\nabla_{g} u\right|_{g}^{2}+|u|^{p+1}\right) d x_{g} d t \\
& +C \int_{0}^{T} \int_{\Omega\left(R_{0}-\varepsilon_{0}\right)}|u|^{2} d x_{g} d t .
\end{aligned}
$$

The estimate (5.2) holds true.

Lemma 5.3. Let assumption (B), assumption (U1) and assumption (U2) hold true and let $T$ be sufficiently large. Then for any $\left\|u_{0}\right\|_{L^{2}(\Omega)} \leq E_{0}$, there exists positive constant $C\left(E_{0}, T\right)$ such that

$$
E(0)+\int_{0}^{T} E(t) d t \leq C\left(E_{0}, T\right) \int_{0}^{T} \int_{\Omega} a(x)\left(|u|^{2}+\left|\nabla_{g} u\right|_{g}^{2}+|u|^{p+1}\right) d x_{g} d t .
$$

Proof. We apply compactness-uniqueness arguments to prove the conclusion. It follows from (5.2) that

$$
\begin{aligned}
E(0)+\int_{0}^{T} E(t) d t \leq & C \int_{0}^{T} \int_{\Omega} a(x)\left(|u|^{2}+\left|\nabla_{g} u\right|_{g}^{2}+|u|^{p+1}\right) d x_{g} d t \\
& +C \int_{0}^{T} \int_{\Omega\left(R_{0}-\varepsilon_{0}\right)}|u|^{2} d x_{g} d t .
\end{aligned}
$$

Then, if the estimate (5.14) doesn't hold true, there exist $\left\{u_{k}\right\}_{k=1}^{\infty}$ such that

$$
\int_{0}^{T} \int_{\Omega\left(R_{0}-\varepsilon_{0}\right)}\left|u_{k}\right|^{2} d x_{g} d t \geq k \int_{0}^{T} \int_{\Omega} a(x)\left(\left|u_{k}\right|^{2}+\left|\nabla_{g} u_{k}\right|_{g}^{2}+\left|u_{k}\right|^{p+1}\right) d x_{g} d t .
$$

Thus,

$$
E_{k}(0)+\int_{0}^{T} E_{k}(t) d t \leq C E_{0}
$$


where

$$
E_{k}(t)=\frac{1}{2} \int_{\Omega}\left(\left|u_{k}\right|^{2}+\left|\nabla_{g} u_{k}\right|_{g}^{2}\right) d x_{g}+\frac{1}{p+1} \int_{\Omega}\left|u_{k}\right|^{p+1} d x_{g}
$$

Therefore, there exists $\hat{u}_{0}$ and a subset of $\left\{u_{k}\right\}_{k=1}^{\infty}$, still denoted by $\left\{u_{k}\right\}_{k=1}^{\infty}$, such that

$$
u_{k} \rightarrow \hat{u}_{0} \text { weakly in } L^{2}\left([0, T], H_{\Gamma}^{1}(\Omega)\right)
$$

and

$$
u_{k} \rightarrow \hat{u}_{0} \text { strongly in } L^{2}\left(\Omega\left(R_{0}-\varepsilon_{0}\right)\right) \text { for arbitrarily fixed } t \in[0, T] \text {. }
$$

Note that

$$
\left\|u_{k}-\hat{u}\right\|_{L^{2}\left(\Omega\left(R_{0}-\varepsilon_{0}\right)\right)}^{2} \leq \widehat{C}(T) E_{0}, \quad \forall t \in[0, T], \forall 1 \leq k<+\infty .
$$

Lebesgue's dominated convergence theorem yields

$$
u_{k} \rightarrow \hat{u}_{0} \text { strongly in } L^{2}\left(\Omega\left(R_{0}-\varepsilon_{0}\right) \times(0, T)\right) \text {. }
$$

\section{Case a:}

$$
\int_{0}^{T} \int_{\Omega\left(R_{0}-\varepsilon_{0}\right)}\left|\hat{u}_{0}\right|^{2} d x_{g} d t>0
$$

It follows from (1.11), (4.1), (4.2) and (5.17) that there exists $C(T)>0$ such that

$$
E_{k}(t) \leq C(T) E_{0}, \quad \forall 0 \leq t \leq T
$$

Denote

$$
q=\frac{2 n}{(n-2) p}, \quad q^{*}=\frac{q}{q-1} .
$$

Since $1<p<\frac{n+2}{n-2}$, then

$$
\frac{2 n}{n+2}<q, q^{*}<\frac{2 n}{n-2}
$$

Note that

$$
\frac{1}{q}+\frac{1}{q^{*}}=1
$$

then, $L^{q^{*}}\left(\Omega\left(R_{0}-\varepsilon_{0}\right)\right)$ is the dual space of $L^{q}\left(\Omega\left(R_{0}-\varepsilon_{0}\right)\right)$.

Note that

$$
H_{\Gamma}^{1}\left(\Omega\left(R_{0}-\varepsilon_{0}\right)\right) \hookrightarrow L^{\frac{2 n}{n-2}}\left(\Omega\left(R_{0}-\varepsilon_{0}\right)\right) .
$$

therefore, it follows from (5.24) that

$$
\left\{\left|u_{k}\right|^{p-1} u_{k}\right\} \text { are bounded in } L^{\infty}\left([0, T], L^{q}\left(\Omega\left(R_{0}-\varepsilon_{0}\right)\right)\right) \text {. }
$$

Then

$$
\left\{\left|u_{k}\right|^{p-1} u_{k}\right\} \text { are bounded in } L^{q}\left(\Omega\left(R_{0}-\varepsilon_{0}\right) \times(0, T)\right) \text {. }
$$

Hence, there exists a subset of $\left\{u_{k}\right\}_{k=1}^{\infty}$, still denoted by $\left\{u_{k}\right\}_{k=1}^{\infty}$, such that

$$
\left|u_{k}\right|^{p-1} u_{k} \rightarrow\left|\hat{u}_{0}\right|^{p-1} \hat{u}_{0} \text { weakly in } L^{q}\left(\Omega\left(R_{0}-\varepsilon_{0}\right) \times(0, T)\right) \text {. }
$$


It follows from (5.16) that

$$
a(x) \hat{u}_{0}=0 \quad(x, t) \in \Omega \times(0, T) .
$$

Therefore, with (5.19) and (5.31), we obtain

$$
\left\{\begin{array}{l}
i \hat{u}_{0 t}+\Delta_{g} \hat{u}_{0}-\left|\hat{u}_{0}\right|^{p-1} \hat{u}_{0}=0 \quad(x, t) \in\left(\Omega\left(R_{0}-\varepsilon_{0}\right) \times(0, T)\right), \\
a(x) \hat{u}_{0}=0 \quad(x, t) \in \Omega \times(0, T) .
\end{array}\right.
$$

With (5.1) and Assumption (U2), we have

$$
\hat{u}_{0} \equiv 0, \quad(x, t) \in \Omega \times(0, T),
$$

which contradicts (5.23).

\section{Case b:}

$$
\hat{u}_{0} \equiv 0 \quad \text { on } \quad \Omega\left(R_{0}-\varepsilon_{0}\right) \times(0, T)
$$

Denote

$$
v_{k}=u_{k} / \sqrt{c_{k}} \quad \text { for } \quad k \geq 1
$$

where

$$
c_{k}=\int_{0}^{T} \int_{\Omega\left(R_{0}-\varepsilon_{0}\right)}\left|u_{k}\right|^{2} d x_{g} d t .
$$

Then $v_{k}$ satisfies

$$
\left\{\begin{array}{l}
i v_{k t}+\Delta_{g} v_{k}+i a(x) v_{k}-\left|u_{k}\right|^{p-1} v_{k}=0 \quad(x, t) \in \Omega \times(0, T), \\
\left.v_{k}\right|_{\Gamma}=0 \quad t \in(0, T),
\end{array}\right.
$$

and

$$
\int_{0}^{T} \int_{\Omega\left(R_{0}-\varepsilon_{0}\right)}\left|v_{k}\right|^{2} d x_{g} d t=1
$$

It follows from (5.16) that

$$
1 \geq k \int_{0}^{T} \int_{\Omega} a(x)\left(\left|v_{k}\right|^{2}+\left|\nabla_{g} v_{k}\right|_{g}^{2}+\left|u_{k}\right|^{p-1}\left|v_{k}\right|^{2}\right) d x_{g} d t .
$$

Therefore, it follows from (5.15) that

$$
\widehat{E}_{k}(0)+\int_{0}^{T} \widehat{E}_{k}(t) d t \leq 1+\frac{1}{k} \leq 2,
$$

where

$$
\widehat{E}_{k}(t)=\int_{\Omega}\left(\left|v_{k}\right|^{2}+\left|\nabla_{g} v_{k}\right|_{g}^{2}+\left|u_{k}\right|^{p-1}\left|v_{k}\right|^{2}\right) d x_{g}
$$

Hence, there exists $v_{0}$ and a subset of $\left\{v_{k}\right\}_{k=1}^{\infty}$, still denoted by $\left\{v_{k}\right\}_{k=1}^{\infty}$, such that

$$
v_{k} \rightarrow v_{0} \text { weakly in } L^{2}\left([0, T], H_{\Gamma}^{1}(\Omega)\right)
$$


and

$$
v_{k} \rightarrow \hat{v}_{0} \text { strongly in } L^{2}\left(\Omega\left(R_{0}-\varepsilon_{0}\right)\right) \text { for arbitrarily fixed } t \in[0, T] \text {. }
$$

Then by Lebesgue's dominated convergence theorem, we obtain

$$
v_{k} \rightarrow v_{0} \text { strongly in } L^{2}\left(\Omega\left(R_{0}-\varepsilon_{0}\right) \times(0, T)\right) .
$$

It follows from (1.11), (4.1) and (4.2) that there exists $C(T)>0$ such that

$$
E_{k}(t) \leq C(T) E_{k}(0), \quad \forall 0 \leq t \leq T
$$

With (5.36) and (5.41), we obtain

$$
\widehat{E}_{k}(t) \leq C(T) \widehat{E}_{k}(0) \leq 2 C(T), \quad \forall 0 \leq t \leq T .
$$

Let $q, q^{*}$ be given by (5.25). Note that

$$
H_{\Gamma}^{1}\left(\Omega\left(R_{0}-\varepsilon_{0}\right)\right) \hookrightarrow L^{\frac{2 n}{n-2}}\left(\Omega\left(R_{0}-\varepsilon_{0}\right)\right) .
$$

Therefore, it follows from (5.47) that

$$
\left\{\left|v_{k}\right|^{p-1} v_{k}\right\} \text { are bounded in } L^{\infty}\left([0, T], L^{q}\left(\Omega\left(R_{0}-\varepsilon_{0}\right)\right)\right) \text {. }
$$

Hence

$$
\begin{aligned}
\int_{0}^{T} \int_{\Omega\left(R_{0}-\varepsilon_{0}\right)} & \left(\left|u_{k}\right|^{p-1}\left|v_{k}\right|\right)^{q} d x_{g} d t \\
& =c_{k}^{\frac{q(p-1)}{2}} \int_{0}^{T} \int_{\Omega\left(R_{0}-\varepsilon_{0}\right)}\left|v_{k}\right|^{\frac{2 n}{n-2}} d x_{g} d t \\
& \leq c_{k}^{\frac{q(p-1)}{2}} C(T) .
\end{aligned}
$$

With (5.35), (5.37) and (5.45), we obtain

$$
\lim _{k \rightarrow+\infty} \int_{0}^{T} \int_{\Omega\left(R_{0}-\varepsilon_{0}\right)}\left(\left|u_{k}\right|^{p-1}\left|v_{k}\right|\right)^{q} d x_{g} d t=0 .
$$

It follows from (5.40) that

$$
a(x) v_{0}=0 \quad(x, t) \in \Omega \times(0, T) .
$$

Therefore, it follows from (5.38), (5.43) and (5.51) that

$$
\left\{\begin{array}{l}
i v_{0 t}+\Delta_{g} v_{0}=0 \quad(x, t) \in \Omega\left(R_{0}-\varepsilon_{0}\right) \times(0, T), \\
a(x) v_{0}=0 \quad(x, t) \in \Omega \times(0, T) .
\end{array}\right.
$$

With (5.1) and Assumption (U1), we have

$$
v_{0} \equiv 0, \quad(x, t) \in \Omega \times(0, T) .
$$


It follows from (5.39) that

$$
\int_{0}^{T} \int_{\Omega\left(R_{0}-\varepsilon_{0}\right)}\left|v_{0}\right|^{2} d x_{g} d t=1
$$

which contradicts (5.54).

Proof of Theorem 2.2 Let $T$ be sufficiently large. It follows from (4.1) that $\|u\|_{L^{2}(\Omega)}$ is non increasing. Hence, with (5.14), we obtain

$$
\begin{aligned}
& E(S)+\int_{S}^{S+T} E(t) d t \\
& \quad \leq C\left(E_{0}, T\right) \int_{S}^{S+T} \int_{\Omega} a(x)\left(|u|^{2}+\left|\nabla_{g} u\right|_{g}^{2}+|u|^{p+1}\right) d x_{g} d t,
\end{aligned}
$$

for any $S \geq 0$.

It follows from (4.1) and (4.2) that

$$
\left.\int_{\Omega}|u|^{2} d x_{g}\right|_{S} ^{S+T}=-2 \int_{S}^{S+T} \int_{\Omega} a(x)|u|^{2} d x_{g} d t
$$

and

$$
\begin{aligned}
\left.\int_{\Omega}\left(\left|\nabla_{g} u\right|_{g}^{2}+\frac{2}{p+1}|u|^{p+1}\right) d x_{g}\right|_{S} ^{S+T} & =-2 \int_{S}^{S+T} \int_{\Omega} a(x)\left(\left|\nabla_{g} u\right|_{g}^{2}+|u|^{p+1}\right) d x_{g} d t \\
& +\int_{S}^{S+T} \int_{\Omega}|u|^{2}\left(\Delta_{g} a(x)\right) d x_{g} d t .
\end{aligned}
$$

Therefore, with (5.56), we deduce that

$$
\begin{aligned}
E(S)+ & \int_{S}^{S+T} E(t) d t \\
\leq & C\left(E_{0}, T\right)(E(S)-E(S+T))+C\left(E_{0}, T\right) \int_{S}^{S+T} \int_{\Omega}\left|\Delta_{g} a(x)\right||u|^{2} d x_{g} d t \\
= & C\left(E_{0}, T\right)(E(S)-E(S+T))-\left.M \int_{\Omega}|u|^{2} d x_{g}\right|_{S} ^{S+T} \\
& +\int_{S}^{S+T} \int_{\Omega}\left(C\left(E_{0}, T\right)\left|\Delta_{g} a(x)\right|-M a(x)\right)|u|^{2} d x_{g} d t
\end{aligned}
$$

For sufficiently large $M$, with (2.28) we have

$$
E(S) \leq C\left(E_{0}, T\right)(E(S)-E(S+T))-\left.M \int_{\Omega}|u|^{2} d x_{g}\right|_{S} ^{S+T} .
$$

Denote

$$
\widetilde{E}(t)=E(t)+\frac{M}{C\left(E_{0}, T\right)} \int_{\Omega}|u|^{2} d x_{g}
$$

From (5.60), we obtain

$$
\widetilde{E}(S) \leq \widetilde{C}\left(E_{0}, T\right)(\widetilde{E}(S)-\widetilde{E}(S+T))
$$


Then

$$
\widetilde{E}(S+T) \leq \frac{\widetilde{C}\left(E_{0}, T\right)-1}{\widetilde{C}\left(E_{0}, T\right)} \widetilde{E}(S) .
$$

It follows from (1.11), (4.1), (4.2) and (5.61) that there exists $\widetilde{C}(T)>0$ such that

$$
\widetilde{E}(S+t) \leq \widetilde{C}(T) \widetilde{E}(S), \quad \forall 0 \leq t \leq T .
$$

With (5.63), $\widetilde{E}(t)$ is of exponential decay. Hence, there exist $C_{1}\left(E_{0}\right), C_{2}\left(E_{0}\right)>0$ such that

$$
E(t) \leq C_{1}\left(E_{0}\right) e^{-C_{2}\left(E_{0}\right) t} E(0), \forall t>0
$$

\section{Proofs of stability with uniform energy decay rate}

Lemma 6.1. Let Assumption (C) hold true. Assume that

$$
\frac{\partial r}{\partial \nu} \leq 0, \quad x \in \Gamma
$$

Let $u(x, t)$ solve the system (1.9). Then

$$
E(0)+\int_{0}^{T} E(t) d t \leq C \int_{0}^{T} \int_{\Omega} a(x)\left(|u|^{2}+\left|\nabla_{g} u\right|_{g}^{2}+|u|^{p+1}\right) d x_{g} d t,
$$

for sufficiently large $T$.

Proof. Let $b(z)$ be a smooth nonnegative function defined on $[0,+\infty)$ satisfying

$$
b(z)=1, \quad 0 \leq z \leq R_{0}-\varepsilon_{0} \quad \text { and } \quad b(z)=0, \quad z \geq R_{0} .
$$

Let

$$
H(x)=b(r) x, \quad x \in \mathbb{R}^{n} .
$$

It follows from (2.34), (2.35) and (3.11) that

$$
\begin{aligned}
& D H(X, X) \geq \delta|X|_{g}^{2} \text { for all } X \in \mathbb{R}_{x}^{n}, x \in \Omega\left(R_{0}-\varepsilon_{0}\right), \\
& \begin{aligned}
\operatorname{div}_{g} H & =1+r \Delta_{g} r \\
& =1+r\left(\frac{n-1}{r}+\frac{\partial \ln \sqrt{\operatorname{det}(G(x))}}{\partial r}\right) \\
& =n+d / 2 \text { for all } X \in \mathbb{R}_{x}^{n}, x \in \Omega\left(R_{0}-\varepsilon_{0}\right),
\end{aligned}
\end{aligned}
$$


Let $\mathcal{H}=H$ and $\widehat{\Omega}=\Omega\left(R_{0}\right)$ in (3.2). From (3.2), we have

$$
\begin{aligned}
\int_{0}^{T} \int_{\Gamma} & \operatorname{Re}\left(\frac{\partial u}{\partial \nu} \mathcal{H}(\bar{u})\right) d \Gamma_{g} d t \\
& +\frac{1}{2} \int_{0}^{T} \int_{\Gamma}\left(\operatorname{Im}\left(u \bar{u}_{t}\right)-\left|\nabla_{g} u\right|_{g}^{2}-\frac{2}{p+1}|u|^{p+1}\right)\langle\mathcal{H}, \nu\rangle_{g} d \Gamma_{g} d t \\
\geq \frac{1}{2} & \left.\int_{\Omega\left(R_{0}\right)} \operatorname{Im}(u H(\bar{u})) d x_{g}\right|_{0} ^{T}+\delta \int_{0}^{T} \int_{\Omega\left(R_{0}-\varepsilon_{0}\right)}\left|\nabla_{g} u\right|_{g}^{2} d x_{g} d t \\
& -C \int_{0}^{T} \int_{x \in \Omega\left(R_{0}\right) \backslash \Omega\left(R_{0}-\varepsilon_{0}\right)}\left|\nabla_{g} u\right|_{g}^{2} d x_{g} d t+\int_{0}^{T} \int_{\Omega\left(R_{0}\right)} \operatorname{Im}(a(x) u H(\bar{u})) d x_{g} d t \\
& +\frac{1}{2} \int_{0}^{T} \int_{\Omega\left(R_{0}\right)}\left(\operatorname{Im}\left(u \bar{u}_{t}\right)-\left|\nabla_{g} u\right|_{g}^{2}-\frac{2}{p+1}|u|^{p+1}\right) \operatorname{div} g H d x_{g} d t \\
\frac{1}{2} & \left.\int_{\Omega\left(R_{0}\right)} \operatorname{Im}(u H(\bar{u})) d x_{g}\right|_{0} ^{T}+\delta \int_{0}^{T} \int_{\Omega\left(R_{0}-\varepsilon_{0}\right)}\left|\nabla_{g} u\right|_{g}^{2} d x_{g} d t \\
& -C \int_{0}^{T} \int_{x \in \Omega\left(R_{0}\right) \backslash \Omega\left(R_{0}-\varepsilon_{0}\right)}\left|\nabla_{g} u\right|_{g}^{2} d x_{g} d t+\int_{0}^{T} \int_{\Omega\left(R_{0}\right)} \operatorname{Im}(a(x) u H(\bar{u})) d x_{g} d t \\
& +\frac{1}{2} \int_{0}^{T} \int_{\Omega\left(R_{0}\right)}\left(\operatorname{Im}\left(u \bar{u}_{t}\right)-\left|\nabla_{g} u\right|_{g}^{2}-|u|^{p+1}\right) \operatorname{div}{ }_{g} H d x_{g} d t \\
& +\int_{0}^{T} \int_{\Omega\left(R_{0}\right)} \frac{(p-1) \operatorname{div}{ }_{g} H}{2(p+1)}|u|^{p+1} d x_{g} d t .
\end{aligned}
$$

Let $P=\frac{\operatorname{div}_{g} H}{2}$ and $\widehat{\Omega}=\Omega\left(R_{0}\right)$ in (3.4). Substituting (3.4) into (6.7), we obtain

$$
\begin{aligned}
\left.\frac{1}{2} \int_{\Omega\left(R_{0}\right)} \operatorname{Im}(u H(\bar{u})) d x_{g}\right|_{0} ^{T}-\frac{1}{4} \int_{0}^{T} \int_{\Omega\left(R_{0}\right)}|u|^{2} \Delta_{g}(\operatorname{div} g) d x_{g} d t \\
\quad+\int_{0}^{T} \int_{\Omega\left(R_{0}\right)} \operatorname{Im}(a(x) u H(\bar{u})) d x_{g} d t+\delta \int_{0}^{T} \int_{\Omega\left(R_{0}-\varepsilon_{0}\right)}\left|\nabla_{g} u\right|_{g}^{2} d x_{g} d t \\
\quad+\int_{0}^{T} \int_{\Omega\left(R_{0}-\varepsilon_{0}\right)} \frac{(n+d / 2)(p-1)}{2(p+1)}|u|^{p+1} d x_{g} d t \\
\leq C \int_{0}^{T} \int_{\Omega\left(R_{0}\right) \backslash \Omega\left(R_{0}-\varepsilon_{0}\right)}\left(\left|\nabla_{g} u\right|_{g}^{2}+|u|^{p+1}\right) d x_{g} d t+\Pi_{\Gamma},
\end{aligned}
$$

where

$$
\begin{aligned}
\Pi_{\Gamma}=\int_{0}^{T} & \int_{\Gamma} \operatorname{Re}\left(\frac{\partial u}{\partial \nu} H(\bar{u})\right) d \Gamma_{g} d t \\
& +\frac{1}{2} \int_{0}^{T} \int_{\Gamma}\left(\operatorname{Im}\left(u \bar{u}_{t}\right)-\left|\nabla_{g} u\right|_{g}^{2}-\frac{2}{p+1}|u|^{p+1}\right)\langle H, \nu\rangle_{g} d \Gamma_{g} d t \\
& -\frac{1}{2} \int_{0}^{T} \int_{\Gamma}|u|^{2} \frac{\partial P}{\partial \nu} d \Gamma_{g} d t+\int_{0}^{T} \int_{\Gamma} \operatorname{Re}\left(P \bar{u} \frac{\partial u}{\partial \nu}\right) d \Gamma_{g} d t
\end{aligned}
$$

Since $\left.u\right|_{\Gamma}=0$, we obtain $\left.\nabla_{\Gamma_{g}} \bar{u}\right|_{\Gamma}=0$, that is,

$$
\nabla_{g} \bar{u}=\frac{\partial \bar{u}}{\partial \nu} \nu \quad \text { for } \quad x \in \Gamma .
$$


Then, with (6.4), we have

$$
H(\bar{u})=\left\langle H, \nabla_{g} \bar{u}\right\rangle_{g}=\frac{\partial \bar{u}}{\partial \nu}\langle H, \nu\rangle_{g}=r \frac{\partial \bar{u}}{\partial \nu} \frac{\partial r}{\partial \nu} \quad \text { for } \quad x \in \Gamma .
$$

Using the formulas (6.10) and (6.11) in formula (6.9) on the portion $\Gamma$, with (6.1), we obtain

$$
\Pi_{\Gamma}=\frac{1}{2} \int_{0}^{T} \int_{\Gamma} r\left|\frac{\partial u}{\partial \nu}\right|^{2} \frac{\partial r}{\partial \nu} d \Gamma_{g} d t \leq 0 .
$$

Substituting (6.12) into (6.8), we have

$$
\begin{aligned}
& \left.\frac{1}{2} \int_{\Omega\left(R_{0}\right)} \operatorname{Im}(u H(\bar{u})) d x_{g}\right|_{0} ^{T}-\frac{1}{4} \int_{0}^{T} \int_{\Omega\left(R_{0}\right)}|u|^{2} \Delta_{g}\left(\operatorname{div}{ }_{g} H\right) d x_{g} d t \\
& \quad+\int_{0}^{T} \int_{\Omega\left(R_{0}\right)} \operatorname{Im}(a(x) u H(\bar{u})) d x_{g} d t \\
& \quad+\delta \int_{0}^{T} \int_{\Omega\left(R_{0}-\varepsilon_{0}\right)}\left|\nabla_{g} u\right|_{g}^{2} d x_{g} d t+\int_{0}^{T} \int_{\Omega\left(R_{0}-\varepsilon_{0}\right)} \frac{(n+d / 2(p-1)}{(p+1)}|u|^{p+1} d x_{g} d t \\
& \leq C \int_{0}^{T} \int_{\Omega\left(R_{0}\right) \backslash \Omega\left(R_{0}-\varepsilon_{0}\right)}\left(\left|\nabla_{g} u\right|_{g}^{2}+|u|^{p+1}\right) d x_{g} d t .
\end{aligned}
$$

Therefore,

$$
\begin{aligned}
\int_{0}^{T} \int_{\Omega\left(R_{0}-\varepsilon_{0}\right)}\left(|u|^{p+1}+\left|\nabla_{g} u\right|_{g}^{2}\right) d x_{g} d t \leq C(E(0)+E(T)) \\
+C \int_{0}^{T} \int_{\Omega\left(R_{0}\right)} a(x)\left(|u|^{2}+\left|\nabla_{g} u\right|_{g}^{2}+|u|^{p+1}\right) d x_{g} d t .
\end{aligned}
$$

Note that

$$
\int_{0}^{T} \int_{\Omega\left(R_{0}-\varepsilon_{0}\right)}|u|^{2} d x_{g} d t \leq C\left(R_{0}\right) \int_{0}^{T} \int_{\Omega\left(R_{0}-\varepsilon_{0}\right)}\left|\nabla_{g} u\right|_{g}^{2} d x_{g} d t .
$$

Hence

$$
\begin{aligned}
\int_{0}^{T} E(t) d t \leq & C(E(0)+E(T)) \\
& +C \int_{0}^{T} \int_{\Omega} a(x)\left(|u|^{2}+\left|\nabla_{g} u\right|_{g}^{2}+|u|^{p+1}\right) d x_{g} d t .
\end{aligned}
$$

With (4.1) and (4.2), we deduce that

$$
\begin{aligned}
C E(T) \leq & C E(0)+C \int_{0}^{T} \int_{\Omega} a(x)\left(|u|^{2}+\left|\nabla_{g} u\right|_{g}^{2}+|u|^{p+1}\right) d x_{g} d t \\
& +\frac{C}{2} \int_{0}^{T} \int_{\Omega}|u|^{2}\left|\Delta_{g} a(x)\right| d x_{g} d t,
\end{aligned}
$$

and

$$
\begin{aligned}
4 C E(0)= & \int_{0}^{4 C} E(t) d t-\int_{0}^{4 C}(E(t)-E(0)) d t \\
\leq & \int_{0}^{4 C} E(t) d t+4 C \int_{0}^{4 C} \int_{\Omega} a(x)\left(|u|^{2}+\left|\nabla_{g} u\right|_{g}^{2}+|u|^{p+1}\right) d x_{g} d t \\
& +2 C \int_{0}^{T} \int_{\Omega}|u|^{2}\left|\Delta_{g} a(x)\right| d x_{g} d t .
\end{aligned}
$$


Substituting (6.17) and (6.18) into (6.16), for $T>4 C$, with (2.37), we have

$$
E(0)+\int_{0}^{T} E(t) d t \leq C \int_{0}^{T} \int_{\Omega} a(x)\left(|u|^{2}+\left|\nabla_{g} u\right|_{g}^{2}+|u|^{p+1}\right) d x_{g} d t .
$$

The estimate (6.2) holds true.

Proof of Theorem 2.3 From (4.1), (4.2) and (6.2), we deduce that

$$
\begin{aligned}
E(0)+\int_{0}^{T} \quad & E(t) d t \leq C(E(0)-E(T))+C \int_{0}^{T} \int_{\Omega\left(R_{0}-\varepsilon_{0}\right)}\left|\Delta_{g} a(x)\right||u|^{2} d x_{g} d t \\
= & C(E(0)-E(T))-\left.M \int_{0}^{T} \int_{\Omega}|u|^{2} d x_{g} d t\right|_{0} ^{T} \\
& +\int_{0}^{T} \int_{\Omega\left(R_{0}-\varepsilon_{0}\right)}\left(C\left|\Delta_{g} a(x)\right|-M \int_{0}^{T} \int_{\Omega} a(x)\right)|u|^{2} d x_{g} d t .
\end{aligned}
$$

For sufficiently large $M$, with (2.37) we have

$$
E(0) \leq C(E(0)-E(T))-\left.M \int_{0}^{T} \int_{\Omega}|u|^{2} d x_{g} d t\right|_{0} ^{T}
$$

Denote

$$
\widetilde{E}(t)=E(t)+M \int_{0}^{T} \int_{\Omega}|u|^{2} d x_{g} d t .
$$

From (6.21), we obtain

$$
\widetilde{E}(0) \leq \widetilde{C}(\widetilde{E}(0)-\widetilde{E}(T))
$$

Then

$$
\widetilde{E}(T) \leq \frac{\widetilde{C}-1}{\widetilde{C}} \widetilde{E}(0) .
$$

It follows from (1.11), (4.1), (4.2) and (6.22) that there exists $\widetilde{C}(T)>0$ such that

$$
\widetilde{E}(t) \leq \widetilde{C}(T) \widetilde{E}(0), \quad \forall 0 \leq t \leq T .
$$

With (6.24), $\widetilde{E}(t)$ is exponentially decaying. Hence, there exist $C_{1}, C_{2}>0$ such that

$$
E(t) \leq C_{1} e^{-C_{2} t} E(0), \forall t>0 \text {. }
$$

\section{Acknowledgements}

The authors would like to express their gratitude to the editors and reviewers for their valuable comments and helpful suggestions. 


\section{A Appendix: Proofs of Assumption (U1) and Assumption (U2) under a strong geometric condition}

Let $\widehat{\Omega} \subset \mathbb{R}^{n}$ be a bounded domain with smooth boundary and $\omega$ be an open subset of $\widehat{\Omega}$ such that

$$
\omega \supset \bigcup_{x \in \partial \widehat{\Omega}}\{y \in \widehat{\Omega}|| y-x \mid<\xi\}
$$

for some $\xi>0$.

Assume that the origin $O \notin \overline{\widehat{\Omega}}$ and

$$
\begin{gathered}
G(x) \frac{\partial}{\partial r}=\frac{\partial}{\partial r}, \quad x \in \mathbb{R}^{n}, \quad \text { and } \quad \operatorname{det}(G(x))=c_{0} r^{d}, \quad x \in \widehat{\Omega}, \\
\left\langle\left((1-\delta) G(x)+\frac{r}{2} \frac{\partial G(x)}{\partial r}\right) X, X\right\rangle \geq 0 \quad \text { for } \quad X \in \mathbb{R}_{x}^{n}, \quad x \in \widehat{\Omega},
\end{gathered}
$$

where $0<\delta \leq 1, c_{0}>0$ and $d$ are constants.

Remark A.1. It follows from (4.6) and (4.7) that

$$
\begin{aligned}
\frac{(n+d / 2-1)}{r} & =\frac{n-1}{r}+\frac{\partial \ln \sqrt{\operatorname{det}(G(x))}}{\partial r}=\Delta_{g} r=t r D^{2} r \\
& \geq(n-1) \frac{\delta}{r}, \quad x \in \widehat{\Omega} .
\end{aligned}
$$

Then

$$
d \geq 2(n-1)(\delta-1) .
$$

Proposition A.1. There exists $T_{1} \geq 0$ such that for any $T>T_{1}$, the only solution $u$ in $C\left([0, T], H^{1}(\widehat{\Omega})\right)$ to the system

$$
\left\{\begin{array}{l}
i u_{t}+\Delta_{g} u-|u|^{p-1} u=0 \quad(x, t) \in \widehat{\Omega} \times(0, T), \\
u=0 \quad(x, t) \in \omega \times(0, T),
\end{array}\right.
$$

is the trivial one $u \equiv 0$.

Proof. Let $b(x) \in C^{\infty}\left(\mathbb{R}^{n}\right)$ be a nonnegative function satisfying

$$
b(x)=1, \quad x \in \widehat{\Omega} \backslash \omega \quad \text { and } \quad b(x)=0, \quad \mathbb{R}^{n} \backslash \widehat{\Omega} .
$$

Let

$$
H(x)=b(x) x, \quad x \in \mathbb{R}^{n} .
$$

It follows from (6.28), (6.29) and (3.11) that

$$
D H(X, X) \geq \delta|X|_{g}^{2} \quad \text { for } \quad \text { all } \quad X \in \mathbb{R}_{x}^{n}, x \in \widehat{\Omega} \backslash \omega,
$$




$$
\begin{aligned}
\operatorname{div}_{g} H & =1+r \Delta_{g} r \\
& =1+r\left(\frac{n-1}{r}+\frac{\partial \ln \sqrt{\operatorname{det}(G(x))}}{\partial r}\right) \\
& =n+d / 2 \text { for all } X \in \mathbb{R}_{x}^{n}, x \in \widehat{\Omega} \backslash \omega
\end{aligned}
$$

Let $a(x)=0$ in (3.1). Let $\mathcal{H}=H$ in (3.2) and $P=\frac{\operatorname{div}_{g} H}{2}$ in (3.4). Substituting (3.4) into (3.2), we obtain

$$
\begin{aligned}
\frac{1}{2} \int_{\widehat{\Omega}} \operatorname{Im} & \left.(u H(\bar{u})) d x_{g}\right|_{0} ^{T}-\frac{1}{4} \int_{0}^{T} \int_{\widehat{\Omega}}|u|^{2} \Delta_{g}\left(\operatorname{div}{ }_{g} H\right) d x_{g} d t \\
& +\int_{0}^{T} \int_{\widehat{\Omega}} \operatorname{Re}\left(D H\left(\nabla_{g} \bar{u}, \nabla_{g} u\right)\right) d x_{g} d t \\
& +\int_{0}^{T} \int_{\widehat{\Omega}} \frac{(p-1) \operatorname{div}_{g} H}{2(p+1)}|u|^{p+1} d x_{g} d t \\
= & 0 .
\end{aligned}
$$

Then

$$
\begin{aligned}
& \int_{0}^{T} \int_{\widehat{\Omega} \backslash \omega}\left(\left|\nabla_{g} u\right|^{2}+\frac{2}{p+1}|u|^{p+1}\right) d x_{g} d t \\
\leq & C\left|\int_{\widehat{\Omega}} \operatorname{Im}(u H(\bar{u})) d x_{g}\right|_{0}^{T} \mid+C \int_{0}^{T} \int_{\omega}\left(\left|\nabla_{g} u\right|^{2}+|u|^{2}+|u|^{p+1}\right) d x_{g} d t .
\end{aligned}
$$

Hence

$$
\int_{0}^{T} \int_{\widehat{\Omega}}\left(\left|\nabla_{g} u\right|^{2}+\frac{2}{p+1}|u|^{p+1}\right) d x_{g} d t \leq 2 C E(0)
$$

Note that

$$
\int_{0}^{T} \int_{\widehat{\Omega}}|u|^{2} d x_{g} d t \leq C \int_{0}^{T} \int_{\widehat{\Omega}}\left|\nabla_{g} u\right|_{g}^{2} d x_{g} d t
$$

Therefore

$$
\int_{0}^{T} \int_{\widehat{\Omega}}\left(|u|^{2}+\left|\nabla_{g} u\right|^{2}+\frac{2}{p+1}|u|^{p+1}\right) d x_{g} d t \leq 2 C E(0)
$$

which implies

$$
(T-C) E(0) \leq 0 .
$$

The assertion (6.32) holds true.

By a similar proof with Proposition (A.1), the following assertion holds.

Proposition A.2. There exists $T_{1} \geq 0$ such that for any $T>T_{1}$, the only solution $u$ in $C\left([0, T], H^{1}(\widehat{\Omega})\right)$ to the system

$$
\left\{\begin{array}{l}
i u_{t}+\Delta_{g} u=0 \quad(x, t) \in \widehat{\Omega} \times(0, T) \\
u=0 \quad(x, t) \in \omega \times(0, T)
\end{array}\right.
$$

is the trivial one $u \equiv 0$. 


\section{References}

[1] Aloui,L.: Smoothing effect for regularized Schrödinger equation on compact manifolds. Collect. Math., 59(1): 53-62, 2008.

[2] Aloui, L.: Smoothing effect for regularized Schrödinger equation on bounded domains. Asymptotic Anal., 59(3-4): 179-193, 2008.

[3] Aloui, L., Khenissi, M.: Stabilization of Schrödinger equation in exterior domains. ESAIM, Control Optim. Calc. Var., 13(3): 570-579, 2007.

[4] Aloui, L., Khenissi, M.: Boundary stabilization of the wave and Schrödinger equations in exterior domains. Discrete and continuous dynamical systems, 27(3): 919-934, 2010.

[5] Bardos, C., Lebeau, G., Rauch, J.: Sharp sufficient conditions for the observation, control, and stabilization of waves from the boundary. SIAM J. Control Optim., 30(5): 1024-1065, 1992.

[6] Bony, J.F., Häfner, D.: Local energy decay for several evolution equations on asymptotically Euclidean manifolds. Annales Scientifiques de l' école Normale Supérieure, 45(2): 311-335, 2012.

[7] Bortot, C.A., Cavalcanti, M.M.: Asympotic stability for the damped Schrödinger equation on noncompact Riemannian manifolds and exterior domains. Comm. Part. Diff. Equations, 39(9): 1791-1820, 2014.

[8] Bouclet, J.M.: Low frequency estimates and local energy decay for asymptotically Euclidean laplacians. Comm. Part. Diff. Equations, 36: 1239-1286, 2011.

[9] Bouclet, J.M., Tzvetkov, N.: On global Strichartz estimates for non-trapping metrics. J. Funct. Anal., 254: 1661-1682, 2008.

[10] Burq, N.: Décroissance de l'énergie locale de l'équation des ondes pour le problème extérieur et absence de résonance au voisinage du réel. Acta Math., 180(1): 1-29, 1998.

[11] Burq, N.: Semi-classical estimates for the resolvent in nontrapping geometries. Int. Math. Res. Not., 5: 221-241, 2002.

[12] Burq, N.: Global Strichartz estimates for nontrapping geometries: About an article by H. F. Smith and C. D. Sogge. Comm. Partial Differential Equations, 28: 1675-1683, 2003.

[13] Burq, N.: Smoothing effect for Schrödinger boundary value problems. Duke Math. J., 123(2): 403-427, 2004.

[14] Burq, N., Gérard, P., Tzvetkov, N.: On nonlinear Schrödinger equations in exterior domains. [Equations de Schrödinger non linéaires dans des domaines extérieurs.] Annales de l'Institut Henri Poincaré Anal. Non Linéaire, 21: 295-318, 2004.

[15] Burq, N., Gérard, P., Tzvetkov, N.: Strichartz inequalities and the nonlinear Schrodinger equation on compact manifolds. Am. J. Math., 126(3), 569-605, 2004.

[16] Chai, S.G., Liu, K.S.: Observability inequalities for the transmission of shallow shells. Systems Control Lett., 55: 726-735, 2006. 
[17] Cavalcanti, M.M., Corrêa, W.J., Lasiecka, I., Lefler, C.: Well-posedness and uniform stability for nonlinear Schrödingerequations with dynamic/Wentzell boundary conditions. Indiana Univ. Math. J., 65(5): 1445-1502, 2016.

[18] Cavalcanti, M.M., Corrêa, W.J., Domingos Cavalcanti, V.N., Tebou, L.: Wellposedness and energy decay estimates in the Cauchy problem for the damped defocusing Schrödinger equation. J. Differ. Equ., 262(3): 2521-2539, 2017.

[19] Cavalcanti, M.M., Domingos Cavalcanti, V.N., Astudillo Rojas,M. R.: Asymptotic behavior of cubic defocusing Schrödinger equations on compact surfaces. Z. Angew. M ath. Phys., 69(4):100,2018.

[20] Cavalcanti, M.M., Domingos Cavalcanti, V.N., Soriano, J.A., Natali, F.: Qualitative aspects for the cubic nonlinear Schrödinger equations with localized damping: exponential and polynomial stabilization. J. Differential Equations., 248(12): 2955- 2971, 2010 .

[21] Cavalcanti, M.M., Domingos Cavalcanti, V.N., Fukuoka, R., Natali, F.: Exponential stability for the 2-D defocusing Schröinger equation with locally distributed damping. Differential Integral Equations., 22(7-8): 617-636, 2009.

[22] Cazenave, T.: Semilinear Schrödinger Equations, Courant Lecture Notes in Mathematics, vol. 10. New York University, Courant Institute of Mathematical Sciences, New York; American Mathematical Society, Providence, 2003.

[23] Christianson, H.: Dispersive estimates for manifolds with one trapped orbit. Comm. Partial Differential Equations, 33: 1147-1174, 2008.

[24] Ducomet, B.: Decay of solutions of the wave equation outside rough surfaces. Computers Math. Applic., 29: 89-108, 1995.

[25] Dehman, B., Gérard, P., Lebeau, G.: Stabilization and control for the nonlinear Schrödinger equation on a compact surface. Math. Z., 254(4): 729-749, 2006.

[26] Escauriaza L.,Kenig, C. E., Ponce G., Vega L.: The sharp Hardy uncertainty principle for Schröodinger evolutions. Duke Math. J., 155: 163-187, 2010.

[27] Escauriaza, L., Kenig, C. E., Ponce, G., Vega, L.: Uniqueness properties of solutions to Schrödinger equations. Bull. Amer. Math. Soc. (N.S.), 49(3): 415-442, 2012.

[28] Grillakis, M.: On nonlinear Schödinger equations. Comm. Partial Differential Equations, 25: 1827-1844, 2000.

[29] Guillarmou, C.,Hassell, A., Sikora, A.: Resolvent at low energy III: the spectral measure. Trans. Amer. Math. Soc., 365(11): 6103-6148, 2013.

[30] Hassell, A., Tao, T., Wunsch, J.: Sharp Strichartz estimates on non-trapping asymptotically conic manifolds. Amer. J. Math., 128: 963-1024, 2006.

[31] Ionescu, A.D.,Staffilani,G.: Semilinear Schrödinger flows on hyperbolic spaces: scattering in $H^{1}$, Math. Ann., 345: 133-158, 2009.

[32] Ionescu, A.D., Pausader, B., Staffilani,G.: On the global well-posedness of energycritical Schrödinger equations in cuved sapsces, Anal. PDE, 5(4): 705-746, 2012. 
[33] Ionescu, A.D., Kenig C.E.: Uniqueness properties of solutions of Schrödinger equations. J. Funct. Anal., 232(1): 90-136, 2006.

[34] Kenig C.E., Ponce G., Vega L.: On unique continuation for nonlinear Schrödinger equations. Comm. Pure Appl. Math., 56: 1247-1262, 2002.

[35] Khenissi, M., Royer,J.: Local energy decay and the smoothing effect for the damped Schrödinger equation, Anal. PDE, 10(6): 1285-1315, 2017.

[36] Koch, H., Tataru, D.: Dispersive estimates for principally normal pseudodifferential operators. Comm. Pure Appl. Math., 58(2): 217-284, 2005.

[37] Lasiecka, I., Triggiani, R., Zhang, X.: Global uniqueness, observability and stabilization of nonconservative Schrödinger equations via pointwise Carleman estimates. I. $H^{1}(\Omega)$-estimates. J. Inverse Ill-Posed Probl., 12(1): 43-123, 2004.

[38] Lasiecka, I., Triggiani, R., Zhang, X.: Global uniqueness, observability and stabilization of nonconservative Schrödinger equations via pointwise Carleman estimates. II. $L^{2}(\Omega)$-estimates. J. Inverse Ill-Posed Probl., 12(2): 183-231,2004.

[39] Lax, P.D., Morawetz, C.S., Phillips, R.S.: Exponential decay of solutions of the wave equation in the exterior of a star-shaped obstacle. Comm. Pure Appl. Math., 16: 477486, 1963.

[40] Laurent, C.: Global controllability and stabilization for the nonlinear Schrödinger equation on some compact manifolds of dimension 3. SIAM J. Math. Anal., 42(2): 785-832, 2010.

[41] Laurent, C.: Internal control of the Schrödinger equation. Math. Control Relat. Fields, 4(2): 161-186, 2014.

[42] Lions, J. L.: Contrôlabilité Exacte, Stabilization et Perturbations de Systèmes Distribuées. Tom 1. Masson, RMA, 1988.

[43] Melrose, R. B.: Singularities and energy decay in acoustic scattering. Duke Math. J., 46: 43-59, 1979 .

[44] Melrose, R., Zworski, M.: Scattering metrics and geodesic flow at infinity. Invent. Math., 124: 389-436, 1996.

[45] Morawetz, C.S.: The decay of solutions of the exterior initial-boundary value problem for the wave equation. Comm. Pure Appl. Math., 14: 561-568, 1961.

[46] Morawetz, C.S.: Decay for solutions of the exterior problem for the wave equation. Comm. Pure Appl. Math., 28: 229-246, 1975.

[47] Morawetz, C.S., Ralston J.V., Strauss, W.A.: Decay of the solution of the wave equation outside non-trapping obstacles. Comm. on Pure and Applied Mathematics, 30: 447-508, 1977.

[48] Ralston, J.: Solution of the wave equation with localized energy. Comm. on Pure and Applied Mathematics, 22: 807-823, 1969.

[49] Rauch, J.: Local decay of scattering solutions to Schrödingers equation. Commun. Math. Phys., 61: 149-168, 1978. 
[50] Rauch, J., Taylor, M.: Exponential decay of solutions to hyperbolic equations in bounded domains. Indiana Univ. Math. J., 24(1): 79-86, 1974.

[51] Royer, J.: Exponential decay for the Schrödinger equation on a dissipative wave guide. Ann. Henri Poincaré, 16(8): 1807-1836, 2015.

[52] Tataru, D.: Local decay of waves on asymptotically flat stationary space-times. Amer. J. Math., 135(2): 361-401, 2013.

[53] Tsutsumi, Y.: Local energy decay of solutions to the free Schrödinger equation in exterior domains. J. Fac. Sci., Univ. Tokyo, Sect. I A, 31: 97-108, 1984.

[54] Smith, H.F., Sogge, C.D.: Global Strichartz estimates for nontrapping perturbations of the Laplacian. Comm. Partial Differential Equations, 25: 2171-2183, 2000.

[55] Wang, G.S. , Wang, M., Zhang,Y.B.: Observability and unique continuation inequalities for the Schrodinger equation. Journal of the European Mathematical Society, 2017-07, in press.

[56] Yao, P.F.: On the observability inequalities for the exact controllability of the wave equation with variable coefficients. SIAM J. Control Optim., 37: 1568-1599, 1999.

[57] Yao, P.F.: Boundary controllability for the quasilinear wave equation. Appl. Math. Optim. 61: 191-233, 2010.

[58] Yao, P.F.: Modeling and Control in Vibrational and Structural Dynamics: A Differential Geometric Approach. Chapman and Hall/CRC Applied Mathematics and Nonlinear Science Series, CRC Press, Boca Raton, FL, 2011.

[59] Zhang, Z.F., Yao, P.F.: Global smooth solutions of the quasi-linear wave equation with internal velocity feedback. SIAM J. Control Optim., 47: 2044-2077, 2008. 